\title{
A Review of Low Frequency Electromagnetic Wave Phenomena Related to Tropospheric-Ionospheric Coupling Mechanisms
}

\author{
Fernando Simões ${ }^{1}$, Robert Pfaff ${ }^{1}$, Jean-Jacques Berthelier ${ }^{2}$, Jeffrey Klenzing ${ }^{1}$ \\ ${ }^{1}$ NASA/GSFC Heliophysics Science Division, Space Weather Laboratory, 8800 Greenbelt Road, \\ Greenbelt, Maryland, 20771, USA (fernando.a.simoes@nasa.gov; robert.f.pfaff@nasa.gov; \\ jeffrey.klenzing@nasa.gov) \\ ${ }^{2}$ LATMOS/IPSL, UPMC, 4 Place Jussieu, 75005 Paris, France (jean- \\ jacques.berthelier@latmos.ipsl.fr)
}

Corresponding author: email - fernando.a.simoes@nasa.gov; phone - 301286 8436; fax - 301 2861648

\begin{abstract}
Investigation of coupling mechanisms between the troposphere and the ionosphere requires a multidisciplinary approach involving several branches of atmospheric sciences, from meteorology, atmospheric chemistry, and fulminology to aeronomy, plasma physics, and space weather. In this work, we review low frequency electromagnetic wave propagation in the Earthionosphere cavity from a troposphere-ionosphere coupling perspective. We discuss electromagnetic wave generation, propagation, and resonance phenomena, considering atmospheric, ionospheric and magnetospheric sources, from lightning and transient luminous events at low altitude to Alfven waves and particle precipitation related to solar and magnetospheric processes. We review in situ ionospheric processes as well as surface and space weather phenomena that drive troposphere-ionosphere dynamics. Effects of aerosols, water vapor distribution, thermodynamic parameters, and cloud charge separation and electrification processes on atmospheric electricity and electromagnetic waves are reviewed. We also briefly revisit ionospheric irregularities such as spread-F and explosive spread-F, sporadic-E, traveling ionospheric disturbances, Trimpi effect, and hiss and plasma turbulence. Regarding the role of the lower boundary of the cavity, we review transient surface phenomena, including seismic activity, earthquakes, volcanic processes and dust electrification. The role of surface and
\end{abstract}


atmospheric gravity waves in ionospheric dynamics is also briefly addressed. We summarize analytical and numerical tools and techniques to model low frequency electromagnetic wave propagation and solving inverse problems and summarize in a final section a few challenging subjects that are important for a better understanding of tropospheric-ionospheric coupling mechanisms.

Keywords: wave propagation; lightning; ionosphere dynamics; aeronomy; troposphereionosphere coupling.

\section{Introduction}

Atmospheric sciences and its multiple branches have contributed to a progressive, sustained enlightenment of the physical and chemical processes that take place in the atmosphere, ionosphere, and magnetosphere and their response to space weather disturbances. Low frequency electromagnetic waves provide a very useful tool to study the global environment of the Earth from the atmosphere to the magnetosphere and the coupling of the various layers within this region. In this work, we address wave emission and propagation in a framework that brings together atmospheric and space plasma scientists to jointly review research and assess troposphere-ionosphere connections. To this aim, we follow an unconventional reviewing method. We first present a brief explanation of key phenomena germane to this coupling and then address their relevance for the subject, taking into account not only recent developments but also important earlier works.

Since this work is part of a wider, structured effort to bring together current knowledge on troposphere-ionosphere coupling mechanisms, we refer to companion papers or recent reviews whenever appropriate. Barr et al. (2000) have reviewed progress in ELF and VLF wave propagation, namely waves of natural or anthropogenic origin, effects of thunderstorm electrification, lightning and sprites, transient phenomena like sferics and whistlers, Schumann resonance, and disturbances at the lower edge of the ionosphere, such as the Trimpi effect. Singh et al. (2005) have reviewed the electromagnetic coupling between the Earth atmosphere and the space environment, focusing on the DC electric circuit and magnetospheric phenomena. Finally, 
Inan et al. (2010) survey ELF and VLF research on lightning-ionosphere interactions and causative discharges, and discuss the role of optical emissions (sprites, jets, halos) and gamma ray flashes in troposphere-ionosphere coupling.

An outline of this article is as follows. Chapter 2 provides an overview of the most commonly observed waves that are used to assess the atmospheric electric environment and troposphereionosphere coupling. In Chapter 3, we describe the surface-ionosphere cavity/waveguide properties related to wave propagation in the atmosphere and ionosphere, relevant boundary conditions, and electromagnetic sources. Chapter 4 presents analytical and numerical approaches, algorithms, and techniques suitable for wave propagation modeling, including phenomenological atmospheric and ionospheric reference models. In Chapter 5, we discuss electromagnetic wave propagation from a global perspective, emphasizing the contributions to the understanding of the Earth's global electric environment. Finally, in Chapter 6, we summarize the significance of ULF-VLF electromagnetic wave phenomena for the investigation of troposphere-ionosphere coupling mechanisms from complementary perspectives, including not only direct impact in energy balance and instability generation but appropriateness for tropospheric and ionospheric in situ and remote sensing studies.

A note before proceeding: Depending on the field of research, different terminology is used to define the various electromagnetic frequency ranges. In this article, we use the familiar frequency nomenclature used in ionosphere-magnetosphere sciences:

Ultra-Low Frequency (ULF) - Frequencies below $3 \mathrm{~Hz}$, Extra Low Frequency (ELF) - Frequencies between $3 \mathrm{~Hz}$ up to $3 \mathrm{kHz}$, Very Low Frequency (VLF) - Frequencies between 3 kHz and $30 \mathrm{kHz}$.

\section{An Overview of Waves Associated with Tropospheric-Ionospheric Coupling}

A sketch of typical ULF-VLF waves that play an important role in troposphere-ionosphere coupling is presented in Figure 1. Included are hydrodynamic (planetary and gravity) waves, Schumann Resonances and Ionospheric Alfven Resonators, and electromagnetic (sferics, tweeks, whistlers, hiss) waves. 


\subsection{Schumann Resonance}

Earth can be regarded as a nearly conducting sphere, wrapped in a thin dielectric atmosphere that extends up to the ionosphere where the conductivity is also substantial. Two types of electromagnetic resonant modes are possible in the surface-ionosphere cavity/waveguide: (i) ELF longitudinal modes of waves propagating around the planet, usually termed Schumann resonances, and (ii) waveguide VLF transverse modes corresponding to the close to vertical propagation between the surface and the ionosphere. Propagation of low frequency electromagnetic waves within a cavity bounded by two, highly conductive, concentric, spherical shells, similar to that formed by the Earth surface and ionosphere, was first studied by Schumann (1952), and the resonance signatures of the cavity later observed in ELF spectra by Balser and Wagner (1960). Several reviews have been published about Schumann resonance on Earth (Galejs, 1972; Bliokh et al., 1980; Sentman, 1995), including a monograph by Nickolaenko and Hayakawa (2002); Simões et al. (2008a) review Schumann resonance models applied to planetary environments. Because of extensive research in the field, we summarize a few preponderant results for troposphere-ionosphere coupling.

Although other electromagnetic sources may play a role in excitation of Schumann resonance modes, such as transient luminous events, magnetospheric waves or volcanic eruptions (Abbas, 1968; Huang et al., 1999; McNutt and Davis, 2000), lightning triggered within mesoscale convective systems are the dominant contributors of ELF waves in the cavity. Schumann resonance measurements are therefore useful for the investigation of lightning occurrence rates and spatial distribution. The ELF modes of the cavity are due to nearly horizontal wave propagation around the globe in the transverse magnetic mode, i.e., with the wave magnetic field and the propagation vector perpendicular to each other. Lightning intensity and distribution in the cavity control the response of ELF waves; for example, daily and seasonal variations have been reported (Balser and Wagner, 1960; Sátori, 1996), and sprites produce a peculiar enhancement of the Schumann resonance spectrum (Boccippio et al., 1995; Williams et al., 2007). Nickolaenko et al. (1996) use long-term Schumann resonance data to deduce temporal variations of global lightning activity. They also estimate the lightning stroke distance to the receiver by assessing spectral lines relative intensity. Boccippio et al. (1998) use lightning optical detection from orbit to calibrate single station Schumann resonance measurements. 
Specifically, combining conventional magnetic direction-finding techniques and Schumann resonance transient information, they compute the direction and distance of lightning strokes. Triangulation using multiple ground-based receiving stations provides of course more accurate estimates (e.g., Füllekrug and Fraser-Smith, 1996) but the single-receiving point concept is quite useful for satellite measurements.

Monitoring of Schumann resonances over long periods of time contributes to inferring annual and semiannual periodicities (e.g., Nickolaenko et al., 1999; Price and Melnikov, 2004). In addition to variability over daily or a few day periods, deducing long term trends is useful for climate research because of a fundamental connection between lightning and thunderstorm activity. For example, Williams (1992) claims using Schumann resonance as a global tropical thermometer to infer temperature fluctuations in the equatorial regions. Williams et al. (2000) and Williams and Sátori (2004) discuss in great detail the lightning-thunderstorm connection and implications for Schumann resonance spectral morphology, namely the 'chimneys-like' distribution of lightning as a function of local time and Schumann resonance amplitude, combining thermodynamic, hydrological, and geographic records with ELF spectral information.

In addition to lightning, transient luminous events also contribute to the electric processes in the atmosphere. Huang et al. (1999) discuss criteria for sprites and elves based on Schumann resonance observations and claim that ground flashes with positive polarity associated with both sprites and elves enhance Schumann resonance response, triggering an increase of the peak amplitude by several times that of average peak structures, an effect similar to a phenomenon related to strong lightning strokes usually known as Q-burst. Boccippio et al. $(1995,1998)$ report peculiar signatures produced by sprites in Schumann resonance spectra. A significant electric coupling between the troposphere and ionosphere occurs in strong cloud-to-ground lightning, and during troposphere-to-ionosphere (blue jets) and ionosphere-to-troposphere (sprites) discharges (Pasko et al., 2002).

The variability of Schumann resonances is associated to variations of not only electromagnetic sources but also atmosphere properties and cavity boundary conditions. Unlike surface changes that can frequently be neglected, variations of the lower ionosphere, which marks the upper 
boundary of the cavity, produce specific features in the ELF Schumann resonance spectra. Disturbances of the lower ionosphere arise from solar events and energetic solar proton precipitation (Reid, 1986; Roldugin et al., 2003), solar wind-magnetosphere interaction and auroral activity (storms and auroral substorms) as well as from gravity, planetary, and tidal waves or, at a local scale, from lightning and transient luminous events (e.g., ISSI COMPANION PAPERS in gravity waves and TLE's), and even from anthropogenic activities such as stratospheric thermonuclear explosions (Madden and Thompson, 1965). Sentman and Fraser (1991) report evidence for Schumann resonance intensity modulation by the local height of the D-region. Schlegel and Füllekrug (1999) investigate the correlation between sudden ionospheric disturbances induced by particle precipitation and Schumann resonance spectra, and conclude that ELF wave propagation is improved because the electron density profile is sharpened. Schumann resonance amplitude, frequency, and cavity $Q$-factor (damping factors) all often change during solar proton events. Nickolaenko and Hayakawa (2002) present an episode of classical polar cap absorption, where the frequency of the first and second eigenmodes decreases by 0.4 and $0.8 \mathrm{~Hz}$, respectively. Madden and Thompson (1965) report that cavity eigenfrequencies were found to be shifted downwards by about $0.5 \mathrm{~Hz}$ due to high altitude nuclear explosions. The interaction between the solar wind and the ionosphere distorts and modulates (by the solar cycle) the upper boundary and influences cavity eigenfrequencies, which also respond to solar flares (Reid, 1986; Roldugin et al., 2003). Finding solar cycle direct impact in Schumann resonance spectra is therefore unsurprising. Solar effects not only modulate the Schumann resonance spectrum but significantly distort the shape of the cavity. Schumann resonance responds to atmospheric and ionospheric ionization transients during solar flares and solar proton events; Schumann resonance frequency often increases with X-ray flux enhancement and decreases with solar proton rise because ionization produced by photons and protons occurs at different altitudes (Schlegel and Füllekrug, 1999; Rolduguin et al., 2003; De et al., 2010). Additionally, the cavity spherical symmetry is ruined by the action of solar wind, daynight asymmetry, and geomagnetic field, resulting in eigenmodes degeneracy removal (e.g., Nickolaenko and Hayakawa, 2002). Those effects can be used for investigating the upper boundary further, namely lightning effects in the ionosphere. 
The Communications/Navigation Outage Forecasting System (C/NOFS) satellite detected, for the first time, Schumann resonance signatures well beyond the upper boundary of the cavity (Simões et al., 2011). The lowest five Schumann resonance peaks are observed at about 7.9, 14.1, 20.6, 26.8, and $32.9 \mathrm{~Hz}$ and match those of ground measurements. The electric field of the first peak is $\sim 0.3$ and $\sim 0.02 \mu \mathrm{Vm}^{-1} \mathrm{~Hz}^{-1 / 2}$ at about 400 and $850 \mathrm{~km}$ altitude compared to $\sim 0.3$ $\mathrm{mVm}^{-1} \mathrm{~Hz}^{-1 / 2}$ measured on the ground. The Schumann resonance signatures are typically detected by C/NOFS when the following conditions are observed: (i) nighttime; (ii) smooth plasma; (iii) low altitude; (iv) little hiss; (v) flying over intense lightning regions. Additionally, Schumann resonance signatures are not detected on the component of the electric field parallel to the geomagnetic field, $\mathrm{B}_{\mathrm{o}}$; for the components perpendicular to $\mathrm{B}_{0}$, the field amplitudes of the zonal (east-west) and meridional (vertical) components are similar. The relevance of these findings for the investigation troposphere-ionosphere coupling mechanisms is unquestionable despite the cavity leakage mechanism is poorly understood. Specifically, combination of in situ and remote sensing ELF electric and magnetic field measurements offers new capabilities to investigate wave propagation in the Earth-ionosphere cavity.

Although large scale significant variations of surface conductivity cannot be expected, earthquakes could possibly change the local atmospheric conductivity and subsequently may produce specific signatures in the Schumann resonance spectrum. From ELF magnetic field data recorded in Japan, Hayakawa et al. (2005) reported an anomalous effect in the $4^{\text {th }}$ harmonic, possibly associated to the Chi-chi earthquake in Taiwan. They observe an anomalous increase of

the amplitude of this $4^{\text {th }}$ eigenmode about a week before the main shock. A similar anomalous feature also appears before the second earthquake, which occurs six weeks later. According to these authors, atmospheric conductivity variations over Taiwan could enhance ELF wave scattering and modify the SR spectrum. Since lightning distribution also affects the highest harmonics of the SR spectrum, it is difficult to firmly establish the exact role of earthquake precursors and more extensive statistical studies seems necessary to ascertain such an effect.

\subsection{Ionospheric Alfvén Resonator}

Alfvén waves are low frequency waves, usually below the ion cyclotron frequency, that occur in an ionized fluid permeated by a magnetic field such as the Earth ionosphere or magnetosphere. 
Plasma disturbances, ion displacement and a restoring magnetic field tension to balance particles inertia lead to oscillation under the form of magnetohydrodynamic (MHD) waves. When the wave vector is parallel to the background magnetic field, Alfvén waves are transverse and termed shear Alfvén modes; if the wave vector is perpendicular to the background magnetic field, Alfvén waves are longitudinal and known as magnetosonic modes. Plasma density heterogeneities from local to global scale in the ionosphere and magnetosphere allow for the formation of waveguides and resonators for shear and magnetosonic Alfvén waves. The ionospheric magnetosonic waveguide results from the nearly total reflection of magnetosonic wave reflection near the ionospheric F-region peak, and waves can be guided thousands of kilometers in the ionosphere due to such a ionospheric ducting (e.g., Greifinger and Greifinger, 1973).

The ionospheric Alfvén resonator results from wave trapping in the vertical direction between the lower boundary of the ionosphere and the magnetosphere (e.g., Polyakov and Rapoport, 1981; Belyaev et al., 1990; Lysak, 1999). Although Alfvén waves play a significant role in a variety of plasma environments, namely the Sun, interstellar medium, compact astrophysical objects, Earth magnetosphere, and even nuclear fusion reactors (e.g., Allan and Poulter, 1992; Narain and Ulmschneider, 1996; Shukla and Stenflo, 1999; Tsurutani and Ho, 1999; Stasiewicz et al., 2000; Elmegreen and Scalo, 2004), we merely discuss results relevant for ionosphere and troposphere-ionosphere coupling mechanisms research. Comprehensive discussions on the physics of Alfvén waves can be found in various monographs and reviews (e.g., Cross, 1988; Gekelman, 1999; Roberts, 2000; Cramer, 2001; Stasiewicz et al., 2000; Tsurutani et al., 2006). A complementary review of the ionospheric Alfvén resonator and ionospheric waveguide, can be found in a companion paper by (ISSI COMPANION PAPER IN ULF waves). Geomagnetic pulsations are frequently associated to Alfvén waves. In this paper we focus our attention in Pc1Pc2 waves, associated with the ionospheric waveguide and ionospheric Alfvén resonator (IAR), and disregard waves with longer wavelengths (Pc3-Pc5), which are mainly relevant for magnetospheric research. Stasiewicz et al. (2000) present a comprehensive review of Alfvén wave models and measurements, including magnetosonic, IAR, and field line resonance results.

Since the Alfvén index of refraction shows in the F-region, magnetosonic wave propagation in the ionospheric waveguide may provide some insight into the F region dynamics. Shvartsburg 
and Stenflo (1993) outline methods for assessing waveguide asymmetry, e.g., day-night and north-south dichotomy. Neudegg et al. (2000) estimate ULF wave attenuation in the waveguide at high latitudes. The later measured geomagnetic pulsations in Antarctica, finding Pc1-Pc2 wave phase velocity and attenuation in the range 300-800 $\mathrm{kms}^{-1}$ and $10-95 \mathrm{dBMm}^{-1}$, respectively, with a corresponding damping of $310 \pm 220 \mathrm{~km}$. Combining measurements from stations in Antarctica and satellites, Hansen et al. (1991) analyze high latitude unstructured Pc1 waves generated in the vicinity of the dayside auroral oval, obtaining different spectral morphology in the morning and afternoon sectors. They interpret Pcl emissions as resulting from ion cyclotron waves generated in the equatorial region, entering the ionosphere within the auroral oval, and then propagating in the ionospheric waveguide to the stations. Chisham and Orr (1997) perform a statistical study of Pc5 emissions observed at mid latitudes and observed similar local time asymmetry between the morning and afternoon sectors. The asymmetry is possibly related to the fact that field line resonances driven by the solar wind interaction with the magnetosphere occurs at higher latitudes during the morning, and the most likely energy source is the Kelvin-Helmholtz instability at the magnetopause boundary. Allan and Wright (1997) develop the nonlinear Kelvin-Helmholtz instability further in the context of field line resonance and ionosphere-magnetosphere coupling.

Alfvén waves can propagate in the ionosphere not only horizontally between the northern and southern hemispheres but also in the radial direction. The magnetosonic mode is sensitive to ionospheric regional and global latitudinal variation while the Alfvén shear mode is more constrained by vertical local variations, thus both types of waves give access to different variation patterns in the ionosphere. As a matter of fact, IAR signatures contribute to constrain electron and ion density profiles between 100-200 km and about one Earth radius. Since propagation in the Alfvén shear mode requires a vertical component of the magnetic field, detection of IAR signatures is more likely at high latitudes. Belyaev et al. (1990) present convincing data of the IAR signature that confirm theoretical predictions; magnetic field data are recorded in the late afternoon, at mid latitude in Gorky, Ukraine and reveal a few peaks in the Pc1 range with $\mathrm{Q} \sim 10$ (Q-factor is a dimensionless quantity related to wave attenuation and peak resolvability), resulting from $\sim 15$ min data averaging. Belyaev et al. (1999) show spectral resonance structures associated to IAR measured at high latitude (Kilpisjärvi, Finland). Measurements of magnetic field with linear and circular polarization show multiple (usually 3 or 
4) peaks, where difference between consecutive peaks varies between $\sim 0.5$ and $1.5 \mathrm{~Hz}$, preferentially with right-circular or linear polarization. Less persuasive measurements, made onboard the Freja satellite at polar latitudes, have been also reported (Grzesiak, 2000). Bösinger et al. (2004) report IAR signatures from measurements recorded in Crete, Greece. During nighttime, spectrograms show up to 20 resolved lines in the frequency range $0.1-4 \mathrm{~Hz}$ and $\sim 0.2$ $\mathrm{Hz}$ between consecutive peaks. In those spectrograms, frequency systematically increases from a fraction of $1 \mathrm{~Hz}$ at sunset up to 3-4 Hz about midnight, an indication of the major changes of the ionospheric profile in the pre-midnight sector. Using the High Frequency Active Auroral Research Program (HAARP), Parent et al. (2010) investigate the effects of substorm dynamics on IAR. Before substorm onset, the spectral resonances display signatures in agreement with the expected variations of the ionosphere. At substorm onset, the IAR signatures disappear either buried in background noise or because the resonance ceases. The authors argue that an increase of the F-region density associated with soft electron precipitation explain the observations because, after the substorm, the signatures reappear and harmonics are shifted to lower frequencies with tighter frequency spacing.

The most pertinent results for investigating troposphere-ionosphere coupling are related to establishing the nature of IAR excitation sources. Surkov et al. (2006) introduce a theory of mid latitude IAR excitation showing that local lightning activity may explain the resonator excitation. Sukhorukov and Stubbe (1997) propose a mechanism to explain IAR excitation by strong lightning discharges followed by transient luminous events. Demekhov et al. (2000) discuss a different approach involving Pc1 waves. It is important mentioning that the frequency range of IAR signatures and Pc1 waves is similar. Therefore, it is important to identify which Pc1 waves could be attributed to IAR phenomena and whether the resonator would act as a filter for Pc1 waves generated elsewhere. High versus low latitude systematic measurements may shed light on IAR excitation mechanisms and whether lightning-related events are relevant for the investigation of troposphere-ionosphere coupling processes.

\subsection{Sferics and Tweeks}

Lightning discharges produce broadband electromagnetic impulses, often termed sferics, that

propagate in the Earth-ionosphere cavity. In addition to the ELF longitudinal modes of the cavity associated with wave propagation around the planet, transverse resonance modes can be excited 
locally in the VLF domain. For an ionospheric effective height of reflection of $75 \mathrm{~km}$, i.e. altitude of the upper boundary, the frequency of the first transverse mode is about $2 \mathrm{kHz}$. Constructive interference resulting from multiple reflections on the surface and ionosphere filter the broadband signal and yield specific waveforms with frequencies related to the height of the upper boundary. When escaping from the cavity and propagating in the ionosphere, possibly along ducts, the signal is slightly dispersed, producing a tweek which is basically a sferic that suffers small frequency dispersion when traveling through the ionosphere. Waves traveling further along geomagnetic field lines through the plasmasphere in the right-handed mode (Storey, 1953), experience larger frequency dispersion and are detected in the conjugate hemisphere as whistlers due to their typical whistling tones. Sferics and tweeks can be used to study the D-region of the ionosphere, tweeks provide information on the E and F-regions, and whistlers depend on the F-region and electron density profiles in the plasmasphere.

Analytical and numerical models have been developed to study VLF wave propagation in the cavity and derive the properties of the electromagnetic source and of the lower ionosphere. Cummer et al. (1998) use a frequency domain sub-ionospheric VLF propagation code to derive nighttime electron density profiles in the D-region and Sukhorukov (1996) develop an analytical model valid for the upper ELF and lower VLF ranges. The tweek waveforms are better resolved and suffer less attenuation at night (typically less than $0.5 \mathrm{~dB} \mathrm{Mm}^{-1}$ as compared to $5 \mathrm{~dB} \mathrm{Mm}^{-1}$ during day-time) due to sharper D-region electron density profiles. When detectable, higher harmonics provide useful information on propagation condition and characteristics of the lower ionosphere (Singh and Singh, 1996; Kumar et al., 2008). Barr et al. (2000) have thoroughly reviewed the major results from experimental studies on sferics, including time and frequency domain methods and triangulation techniques.

Ohya et al. (2006) use tweek atmospherics observations made during a major geomagnetic storm in October 2000 to determine the D-region disturbances. Reeve and Rycroft (1972) use sferics and tweeks to derive the variations of the lower ionosphere during the solar eclipse of 7 March 1970 and show that the effective height of reflection moves from $69 \mathrm{~km}$ before eclipse to $76 \mathrm{~km}$ at the time of total eclipse. From transverse resonance observations during a stratospheric balloon flight in the equatorial region, Simões et al. (2009) report the occurrence of a fast 
depletion of the D-region between 73 and $82 \mathrm{~km}$ after sunset. Since the D-region is rather difficult to explore both with remote sensing and in situ techniques, systematic high resolution measurements of sferics and determination of their sources by lightning location networks provide a suitable tool for monitoring electron density profiles. Rocket-triggered lightning offers even better experimental conditions because the stroke location uncertainty would be considerably reduced.

Electromagnetic transients are useful to assess not only the ionospheric lower boundary but also medium anisotropy. Hayakawa et al. (1994) use sferics and tweek characteristics, namely polarization, incidence angle, and frequency to investigate ionospheric plasma. They find that wave polarization slightly above the transverse mode cutoff frequency is always left-handed and becomes exactly circular when the cutoff is reached, confirming anisotropy role in wave propagation in the ionosphere. Ohya et al. (2006) use tweek atmospherics observations made during a major geomagnetic storm in October 2000 to determine the D-region response under high magnetic activity. Tweek reflection effective height is used to investigate ionosphere dynamics during the storm and dissimilar ionospheric dynamic conditions were identified. Shvets and Hayakawa (1998) assess tweek polarization effects in the northern and southern hemispheres employing multimode analysis, finding non-reciprocity between East-West and West-East propagation and variations in wave polarization due to the geomagnetic field. From measurements of right- and left-hand ELF-VLF polarized waves, Ostapenko et al. (2010) use tweek signatures to study the auroral region. Atmospherics can therefore contribute to assess not only electron density profiles and D-region dynamics but also ionospheric plasma anisotropy. Injection of VLF signals from ground based transmitters nevertheless offer more reliable choices for studying polarization effects and ionosphere anisotropy because electromagnetic source characteristics are well known (e.g., Inan et al. 2007).

Harrison et al. (2010) suggest using tweeks to investigate possible ionospheric disturbances related to seismic activity. Specifically, they propose a mechanism to link seismic activity and changes in the local lower ionosphere that could be tested by measuring the tweeks cutoff frequency. 


\subsection{Whistlers}

Since the wave velocity is a function of the electron density, the dispersion characteristics of whistlers have been used to derive electron density in the plasmasphere. Favorable reflection conditions in the ionosphere may allow successive reflections between conjugate hemispheres with an increased dispersion observed for each travel. Ion cyclotron whistlers, only detectable in space, also provide information on the ion composition (Gurnett et al., 1965). Whistlers have been extensively used because either they are fundamental for a number of ionospheric or magnetospheric processes such as gyroresonant wave-particle interaction (Summers and Ma, 2000; Brautigam and Albert, 2000), electron acceleration and precipitation from radiation belts (Meredith et al., 2003; Horne et al., 2005), non-linear wave-wave interaction with Alfvén waves (Sharma et al., 2010) or they feature a valuable tool to study the structure of the ionized terrestrial environment (e.g., Park et al., 1978) and its dynamics in response to solar wind disturbances (e.g., Meredith et al., 2001). Monographs by Helliwell (1965), Sazhin (1993), and Ferencz et al. (2001) thoroughly cover whistler theory, measurements, and applications. In the following we briefly summarize a few results directly impacting tropospheric-ionospheric coupling.

Low Ionosphere electron density enhancements are produced by sources located in the cavity or outer space. In addition to transitory ionization related to cosmic ray bursts, solar particle events and flares, lightning, transient luminous events, and terrestrial gamma flashes, electron density enhancements can be produced by energetic electron precipitation driven by the interaction of whistlers with trapped particles in the radiation belt. Haldoupis et al. (2004) present a few VLF signals associated with whistler-induced electron precipitation events, which are produced by electron precipitation due to whistler wave injection into the magnetosphere by the same lightning flash that led to a sprite. Lightning also induces direct ionization enhancements in the low ionosphere. Strangeways (1999) discusses effects of D-region local ionization enhancements produced by lightning, namely ducting development associated to whistlers and amplitude perturbations on subionosphere VLF wave propagation (Trimpi effect). Lightning-generated whistlers lead to coupling between the troposphere, ionosphere, and magnetosphere. A lightning stroke can generate whistlers that interact with cyclotron resonant radiation belt electrons, producing particle acceleration and inducing particle precipitation back in the low ionosphere. 
Determining the depth of penetration of precipitating particles is pertinent to investigate ozone and nitrogen oxides chemistry, for example. Rodger et al. (2007) determine the altitude range where particle precipitation plays a role and assess implications for ionization balance and neutrals chemistry in the mesosphere, by contrasting cosmic rays, solar photoionization, and whistler-induced electron precipitation contributions for the ionization budget. Whereas the ionization due to whistler-induced electron precipitation can be neglected during daytime compared to solar EUV and soft X-ray ionization, nighttime conditions offer a more complex situation. Below $\sim 70 \mathrm{~km}$ the cosmic ray contribution prevails but particle precipitation usually dominates in the range $75-80 \mathrm{~km}$. Despite previous research concluding that particle precipitation can lead to large-scale changes in neutrals chemistry and enhance key species that drive ozone loss, studies suggest that transient electron precipitation plays an important role in some parts of the mesosphere but can be neglected in neutrals chemistry models. Horne et al. (2009) and Lam et al. (2010) scrutinize the role of high energy electron precipitation under different geomagnetic activity conditions, observe precipitating flux increasing with geomagnetic activity, and also address possible implications for atmospheric chemistry and climate variability. Interestingly, lightning interacts with the low ionosphere both through direct (fast) and indirect (delayed) processes. Direct heating and ionization are due to charge transfer to and from the ionosphere. Lightning-induced particle precipitation resulting from interaction with whistlers also produces ionization from above. The study by Dowden et al. (1994) details a way to distinguish between the fast and delayed effects of lightning discharges, concluding that rapid onset, rapid decay of phase and amplitude perturbations of VLF subionospheric transmissions are directly related to atmospheric discharging processes rather than whistler-induced electron precipitation.

Employing statistical analyses, Hayakawa et al. (1993) investigate the possible role of seismic activity role in whistler wave generation and/or propagation. They claim that whistlers where dispersion is at least twice the average are likely correlated with seismic activity. Shalimov and Gokhberg (1998) explore the topic further and argue that anomalous impulsive VLF emissions in the upper ionosphere may be related to whistler trapping in ducts, formed in the ionosphere above the seismically active region. 


\subsection{Geomagnetic Pulsations}

Geomagnetic pulsations are hydromagnetic waves that arise from resonant processes and propagate in the magnetosphere, usually observed along closed field lines or close to these regions. These waves cover the ULF-ELF range, from the longest wavelengths that the magnetospheric cavity can sustain up to ion gyrofrequencies. Geomagnetic pulsations were first observed in 1859 during aurora events (Stewart, 1861) and are often split in morphological groups or frequency bands, including Pc1 (0.2-5 s), Pc2 (5-10 s), Pc3 (10-45 s), Pc4 (45-150 s), Pc5 (150-600 s), Pil (1-40 s), and Pi2 (40-150 s), where Pc and Pi denote continuous and irregular pulsations, respectively. These waves result from electromagnetic ion cyclotron instability, compressional fluctuations of the medium, toroidal and poloidal geometry related propagation, field-aligned currents, magnetic field line resonances, as well as from a coupling between multiple mechanisms.

Saito (1969) reviews geomagnetic pulsations and their classification, observations, and the generation mechanisms. This assessment remains a consistent work in the field and was followed by a number of updated reviews on more specific topics such as type of pulsation, frequency range of occurrence, and satellite observations, emphasizing geomagnetic pulsations relevance for a variety of Earth and space science studies (Raspopov and Lanzerotti, 1976; Pilipenko, 1990; Engebretson et al., 1991; Sazhin and Hayakawa, 1994; Takahashi, 1998; Kangas et al., 1998; Daglis et al., 1999; Olson, 1999; Yahnin and Yahnina, 2007; Zong et al., 2009).

Frequency characteristics (e.g., dispersion relation and harmonics structure), polarization, spatial distribution, or correlation with solar wind and geomagnetic activity make geomagnetic pulsations a valuable tool to investigate Sun-Earth connections, solar wind-magnetosphere interaction, magnetosphere dynamics, and auroral processes. Although geomagnetic pulsations play a minor role in troposphere-ionosphere coupling, a few works deserve attention owing to their interesting results.

The ionosphere in the F-region plays a significant role on geomagnetic pulsations controlling their attenuation and propagation and their coupling with ionospheric currents. Hughes and Southwood (1976) address atmospheric and ionospheric screening effects and show that only a small energy fraction of the signal, about $10 \%$, reaches the ground showing that the strong day- 
night asymmetry results from the fact that ionosphere reflects much less at night. Their work also elucidates the morphological differences between typical day Pc and night Pi signatures. Sarma and Sastry (1995) discuss the enhancement of geomagnetic pulsations near the dip equator due to the equatorial electrojet. Results indicate a sharp cutoff at about $20 \mathrm{~s}$, bearing evidence for distinctive contributions in the Pc1-2 and Pc3-5 domains and the role of the equatorial electrojet in hydromagnetic wave propagation.

An interesting contribution of research work on geomagnetic pulsations is related to phenomena associated with seismic activity and lightning. Iyemori et al. (2005) describe a pulsation of period 3.6 min (Pc5) observed in Phimai, Thailand, 12 min after the Sumatra earthquake on 26 December 2004. A 30 s (Pc3) period pulsation was observed in Tong Hai, China, located 10 degrees north of Phimai. They argue that the nature and period of the geomagnetic pulsation was consistent with a dynamo action in the low ionosphere due to gravity waves triggered by the ocean floor displacement. It is also speculated that the Pc3 signal observed in Tong Hai resulted from magnetosonic waves generated by electric and magnetic perturbations of the dynamo current triggered by the earthquake. Although a cause-effect is difficult to establish, this example illustrates the complex interaction between acoustic and electromagnetic waves involving surface, atmospheric, ionospheric, and magnetospheric phenomena. Fraser-Smith (1993) presents ULF magnetic field measurements related to thunderstorm activity and discusses their

significance to geomagnetic pulsation generation. A correlation between magnetic and thunderstorm activity is found, indicating that lightning could possibly generate geomagnetic pulsations.

\section{The Surface-Ionosphere Cavity and Waveguide Characteristics}

\subsection{Boundary Conditions}

Except for detailed studies involving Alfvén waves or localized ionospheric phenomena, the Earth's surface, where the electric conductivity changes by more than 10 orders of magnitude, is considered as the inner boundary. The situation is far more complex for the upper boundary, which is located in the region between 70 and $110 \mathrm{~km}$, where the conductivity increases by 5 to 6 
orders of magnitude, but needs to be defined more precisely in particular as a function of the frequency range of interest.

Depending on the chosen formalism and on the frequency range of interest, the definition of the upper boundary of the cavity and of the investigation of its characteristics usually employs three complementary concepts: (i) propagation, reflection, and transmission coefficients, (ii) refractive index profile and (iii) skin depth. The concept of a conducting layer in the D-region playing the role of the upper boundary is valid for the DC global electric circuit (e.g., Rycroft, 2006), SR (e.g., Sentman, 1995), as well for sferics and tweeks propagation (e.g., Simões et al., 2009). In the case of the DC electric circuit and to a first approximation, the ionosphere is considered equipotential above $\sim 90 \mathrm{~km}$. For ELF wave propagation, the skin depth of propagating waves is considerably smaller than cavity thickness and a similar boundary also applies. The skin depth, defined as the distance at which the signal decays to $1 / \mathrm{e}$ with respect to the reference position, is $\delta \approx \sqrt{2 / \mu \omega \sigma}$, where $\mu, \sigma$, and $\omega$ are the permeability and conductivity of the medium and the angular frequency of the propagating wave, respectively. In the ELF range, the skin depth is lower than $10 \mathrm{~km}$ for an altitude of $100 \mathrm{~km}$, where the upper boundary of the cavity is usually defined. A similar condition can be applied to the propagation of sferics and tweeks.

For ULF waves such as geomagnetic pulsations, the boundary is usually taken at the altitude where the Alfvén index of refraction presents a sharp variation, i.e. where strong reflection occurs. The inner and outer boundaries of the ionospheric waveguide and ionospheric Alfvén resonator are respectively located in the E-region and at several hundred/thousand kilometers above (e.g., Greifinger and Greifinger, 1968; Polyakov and Rapoport, 1981). Unlike the E-region inner boundary that corresponds to an altitude range with strong gradients in the Alfvén index, the position of the outer boundary varies significantly with local time and latitude because the gradient of the Alfvén index of refraction above the F-peak is much smoother. Some models often set the inner boundary at the surface, thus considering wave propagation through the Eregion down to the ground. This may be a reasonable first order assumption since geomagnetic pulsations related the ionospheric Alfvén resonator are detected at ground, implying that the subsurface acts indeed as the lower boundary. 
Unlike the static, sharp inner boundary, the ionosphere defines a fuzzy, heterogeneous, and anisotropic boundary condition. For quasi-static and electromagnetic phenomena modeling, the surface can be considered a steady, perfect electric conductor despite transient events such as earthquakes, tsunamis, and volcanic eruptions induce local atmospheric conductivity modifications or ionospheric disturbances (ISSI COMPANION PAPER in chemistry and aeronomy). Ionosphere dynamics, however, contributes to an intricate outer boundary because of solar wind, magnetospheric, and particle precipitation driving mechanisms from above combined with lightning, transient luminous events, and gravity waves from below. Plasma irregularities occurring in the ionosphere, namely density enhancements and depletions of the medium, extend ionosphere dynamics further (e.g., de La Beaujardière et al. 2004, 2009; Klenzing et al. 2011). As a result, the ionosphere is significantly asymmetric because of the day-night dichotomy and polar heterogeneity, and particularly dynamic due to solar activity, particle precipitation, unsettled geomagnetic field, ionospheric currents, and thunderstorm activity.

\subsection{Medium Parameterization}

The Appleton-Hartree description of plasma waves in cold magnetized plasma is the basis of the description of wave propagation. Depending on the frequency the dispersion relation can be simplified to provide treatable, analytical solutions. In general, medium parameterization includes electron and ion density, geomagnetic field, and collision frequencies. The plasma anisotropy with respect to magnetic field must be included in Alfvén wave and whistler-mode propagation but is often neglected in ELF wave modeling. Often considered simplifications can be summarized as follows:

(i) Alfvén waves: heavy ions and collision frequency are neglected, when included, Pedersen conductivity introduces wave attenuation and Hall conductivity leads to coupling between shear and magnetosonic modes. Analytical approximations frequently employ simplified profiles of the Alfvén velocity with a propagation at constant velocity in the E-region (hundreds of $\mathrm{kms}^{-1}$ ) and F-region (tens of $\mathrm{kms}^{-1}$ ), and at a velocity that increases exponentially (scale height $\sim 200$ km) above the F-peak (e.g., Polyakov and Rapoport, 1981). More realistic models also include propagation below the D-region, allowing propagation down to the surface. 
(ii) Schumann resonances: anisotropy is frequently neglected, leading to a scalar conductivity that increases with altitude. The conductivity profile is often represented as a "knee-model" with two scale heights respectively below and above a transition layer at $\sim 50 \mathrm{~km}$, the altitude where the magnitude of displacement and conduction currents is similar (e.g., Greifinger and Greifinger, 1978). The conductivity variation with latitude is often neglected because the latitudinal gradient in the troposphere and stratosphere is small compared to the gradient in the vertical direction (e.g., Holzworth et al., 1985). In such a case spherically symmetric analytical solutions may be sought (Sentman, 1990). However more subtle effects, such as frequency splitting of harmonics, requires day/night asymmetry and, possibly, polar cap specific conditions to be taken into account, with only numerical solutions available.

(iii) Sferics, tweeks: anisotropy corrections are frequently included in the investigation of sferics and tweeks and heavy ions are sometimes neglected.

(iv) whistlers: anisotropy must be taken into account for solving electron and ion whistler mode propagation and heavy ions are included when ion cyclotron whistlers are considered. Collision frequencies are often neglected.

\subsection{Electromagnetic Sources}

\subsubsection{Sources Within the Cavity}

Lightning. Lightning results from thunderstorm electrification and charge separation mechanisms with typical discharge channel lengths of 1 to $\sim 10 \mathrm{~km}$ and often involving intricate multiple branches that extend to several kilometers in the vertical and horizontal directions. A detailed description of the lightning discharges and physical processes may be found in the monograph by Rakov and Uman (2007). Lightning discharges are usually termed intracloud/intercloud (the most common), cloud-to-ground, and ground-to-cloud (the least common), and involve positive or negative charge transfer through the channel. Typically, negative cloud-to-ground discharges transfer a total of $25 \mathrm{C}$ with a peak current of $\sim 30 \mathrm{kA}$, releasing about $500 \mathrm{MJ}$; positive discharges are less frequent though charge and peak current are sometimes ten times larger. Lightning occurs mostly over land, in the equatorial regions, and follows a regular seasonal pattern reversing between the northern and southern hemispheres. The global lightning average 
rate is $44 \pm 5 \mathrm{~s}^{-1}$, reaching a maximum of $\sim 200$ strokes per square kilometer per year in Central Africa (geographic coordinates $3^{\circ} \mathrm{S}, 28^{\circ} \mathrm{E}$ ). Individual thunderstorms can deliver up to about 6000 strokes per hour (e.g., Rakov and Uman, 2007; Schumann and Huntrieser, 2007). Relevant characteristics of individual lightning involves flash duration, number of return strokes per flash, charge quantity per flash and per stroke, duration and intensity of continuing current, time to peak and return stroke current, etc.

Lightning plays multiple roles in wave propagation because not only generates electromagnetic waves but induces medium properties modification through local ionization. Lightning correlation with water vapor and aerosols, namely clouds, urban pollutants, dust storms, and smoke plumes offers complementary tools for atmospheric sciences research. In general, atmospheric conductivity is a function of aerosol content, hence providing a link to wave propagation. Fitzgerald (1991) reviews the background aerosol in the boundary layer over oceans remote locations to estimate marine and land nitrate contributions to sea salt aerosol budget. Although the associated chemistry processes are recognized, nitrate relative importance of the ocean, stratosphere, and lightning as a source of the nitrogen-containing precursor gases remains unclear. Over land, however, pollution plays a more obvious role in lightning and atmospheric chemistry. From examination of chemical characteristics of continental outflow, Talbot et al. (1996) suggest that, in addition to biomass burning, lightning or recycled reactive nitrogen may be an important source of nitric oxide (NO) to the upper troposphere. Real et al. (2010) analyze implications of biomass burning pollutants for ozone $\left(\mathrm{O}_{3}\right)$ production and conclude that, whilst the transport of pollutants to the upper troposphere is variable, pollution from biomass burning can make a supplementary contribution to photochemical production of $\mathrm{O}_{3}$ in addition to $\mathrm{NO}_{\mathrm{x}}$ from lightning. Bell et al. (2009) analyze extensive US lightning datasets and find evidence for a lightning weekly cycle related to storm invigoration by pollution. The weekly cycle appears to be reduced over population centers. Lightning rate is also enhanced by smoke plumes. As a result of thunderstorms ingesting smoke from forest fires, Lyons et al. (1998) found enhanced positive cloud-to-ground lightning, triple than that of the climatological norm, and peak currents double than expected. They also claim those thunderstorms produced abnormally high number of sprites. Hurricanes are important sources of lightning, too. Khain et al. (2008) discuss possible aerosol effects on lightning activity and structure of hurricanes. Although the mechanism 
responsible for the formation of the maximum flash rate in the periphery is poorly understood, intense and persistent lightning takes place within a $250-300 \mathrm{~km}$ radius ring around the hurricane center.

Nitrogen fixation is essential for ecological systems growth and is accomplished through anthropogenic and natural processes. Among the natural abiogenic processes, lightning assists the conversion of molecular nitrogen to $\mathrm{NO}$, hence contributing to nitrogen fixation. $\mathrm{NO}_{\mathrm{x}}$ also plays a key role in tropospheric and stratospheric photochemistry by acting to control the concentrations of $\mathrm{O}_{3}$ and hydroxyl $(\mathrm{OH})$. Lightning contributes a few percent to the total $\mathrm{NO}_{\mathrm{x}}$ budget but can be a leading mechanism in pollutant-free areas and the upper troposphere. $\mathrm{NO}_{\mathrm{x}}$ radicals can be also produced by ionospheric particle precipitation in the stratosphere and mesosphere. Schumann and Huntrieser (2007) review the global lightning-induced $\mathrm{NO}_{\mathrm{x}}$ sources and the significance for understanding and predicting $\mathrm{O}_{3}$ distribution and trends in the troposphere, the oxidizing capability of the atmosphere, and the lifetime of trace gases destroyed by reactions with $\mathrm{OH}$. They conclude that a typical thunderstorm produces $250 \mathrm{~mol} \mathrm{NO}_{\mathrm{x}}$ per flash (ISSI COMPANION PAPER IN tropospheric chemistry).

Maximum electric fields measured in thunderclouds are $0.1-0.2 \mathrm{MVm}^{-1}$, a fraction of the 1 $\mathrm{MVm}^{-1}$ conventional breakdown. To overcome the lack of electric field strength, two mechanisms are usually invoked for lightning initiation: (i) runaway breakdown initiated by cosmic rays (e.g., Gurevich and Zybin, 2001) and (ii) positive streamers triggered by hydrometeors, i.e., products resulting from atmospheric water vapor condensation (e.g., Petersen et al., 2008). The former reflects a close relationship between cosmic rays and electrodynamic processes in the thunderstorm atmosphere. The latter is closely related to the thermodynamic properties of the convective cell, which can be used to assess the hydrometeor mass (e.g., James and Markowski, 2010). Milikh and Roussel-Dupré (2010) review runaway breakdown and electrical discharges in thunderstorms and discuss observations bearing evidence for the presence of energetic particles in lightning initiation, including gamma-ray and x-ray flux intensification over thunderstorms, gamma-ray and x-ray bursts in conjunction with stepped leaders, terrestrial gamma-ray flashes, and neutrons production. Ebert et al. (2010) review the relevance of streamer discharges for lightning and sprites inception. Because large sprite discharges at the low air 
densities of the mesosphere are physically similar to small streamer discharges in air at standard temperature and pressure, streamers are useful for investigating the tropospheric-ionospheric electric coupling.

As far as electromagnetic waves are concerned, the lightning channel works as an effective transmitter over a wide frequency band, from ULF to UHF with a frequency spectrum peaked at a few to $10 \mathrm{kHz}$. Lightning distribution around the globe as a function of position and time is also preponderant. Lightning characterization in the time and frequency domains is fundamental to model wave propagation in the Earth-Ionosphere cavity. In the harmonic formalism, lightning is usually described by a Hertz dipole with a convenient frequency spectrum. Sometimes, electric tripoles are also considered to mimic lightning low frequency emission because they offer a better representation of charge distribution in the cloud. In the time domain, a time-varying current profile is considered. Several models have been used to compute the return stroke current of lightning (Nucci et al., 1990). Since the first successful lightning return stroke model based on a double exponential expression to facilitate analytical approximations, lightning models have been improved and classified in four major categories by Rakov and Uman (1998): (i) gas dynamics models involving coupling between gas dynamics equations (conservation of mass, momentum, and energy) and equations of state; (ii) electromagnetic models solving Maxwell equations to derive current distribution along the lightning channel, which is considered a thin wire antenna (charge channel diameter seldom exceeds $1 \mathrm{~cm}$ ); (iii) transmission line models using resistor, capacitor, and inductor elements to compute current fields; (iv) phenomenological (engineering) models, where the spatial and temporal distribution of the return stroke current is specified from lightning characteristics. The latter model offers better approaches to compute the electromagnetic field distribution as function of space and time. Depending on relevant wave propagation modes, lightning strokes are frequently simplified by considering fast $(\sim 100 \mu \mathrm{s})$ and slow ( $\sim 10 \mathrm{~ms})$ variation components, corresponding to the return stroke and continuing current contributions.

We now briefly mention a couple of studies where lightning plays multiple roles, emphasizing complementarity perspectives among atmospheric chemistry, meteorology, atmospheric electricity, and electromagnetic wave propagation. Rodriguez et al. (1992b) discuss a case-study 
of lightning, whistlers, and associated ionospheric effects occurred during a particle precipitation event, and Rodriguez et al. (1992a) assess D-region disturbances caused by lightning. In these works, they claim that weak electromagnetic pulses originating from lightning heat D-region electrons; on the other hand, strong electromagnetic pulses would create electron density enhancements. Perturbations in artificial VLF signals originating in the proximity of storm centers are attributed to low ionosphere heating induced by lightning. Price (2000) claims evidence for a link between global lightning activity and upper tropospheric water vapor. A positive correlation between upper troposphere water vapor variability and global lightning activity is found, suggesting that water vapor variability can be estimated from lightning activity and ELF-VLF wave monitoring.

Although marginally relevant for the present review, less familiar types of discharges, namely ball lightning, also occur in the troposphere. The interested reader can find extensive material about this topic in the monograph by Stenhoff (1999). Several theories related to waves in plasma, microwave interference and cavity modes, and transient wave propagation aiming to explain the phenomenon may be of interest.

Volcanic Eruptions. Volcanic eruptions have been known to produce electrostatic and electromagnetic activity (for a review, see Johnston, 1997; Mather and Harrison, 2006). Impact charging plays an important role in the volcanic plume dust particles can get charged with their charges depending on the nature and velocity of the colliding particles, leading to charge separation mechanisms (Aizawa et al., 2010) rather similar to those acting in thunderstorm clouds. A large number of volcanic eruptions have been reported to produce lightning, with stroke rates up to 1 every 3 seconds during the eruption of Mount Spurr in Alaska (McNutt and Davis, 2000). Like in thunderstorms, volcano lightning occurs within the plume (equivalent to $\mathrm{CC}$ discharges) or between plume and ground (equivalent to CG and GC discharges). Lightninglike discharges has been regularly noticed in forest fire ash plumes, too. Depending on natural conditions, namely plume size and velocity, volcano lightning can be almost as intense as in thunderstorms. 
At night, when observation conditions are more favorable, flashes have been seen at least $20 \mathrm{~km}$ far from the eruption, suggesting that significant amounts of energy are involved in the processes. Typically, discharges are hundreds of meters in length, transfer up to 0.1-0.5 C per event, produce local electric fields in the order of several $\mathrm{kVm}^{-1}$, typically release about $10^{6} \mathrm{~J}$, and seem to begin about 20 min after the eruptions vigorous initiation (Anderson et al., 1965; Brook et at., 1974; Katahira, 1992; McNutt and Davis, 2000). The geometric-mean peak currents of either polarity related to volcano lightning were sometimes only a factor of 2-3 lower than those associated to ordinary lightning recorded by the same network. Continuous measurements conducted at Sakurajima volcano, Japan, revealed syneruption (a period characterized by geologically instantaneous production of large volumes of volcaniclastic sediment) electric pulses, sometimes accompanied by geomagnetic pulses and lightning flashes. Pulses were observed more than 10 seconds after the onset of the eruption, and tend to occur during eruptions that emit volcanic ash to high altitudes (Aizawa et al., 2010). From a perspective of atmospheric electricity investigations, namely the global electric circuit and electromagnetic wave propagation, volcanic lightning offers contrasting contributions. On the one hand, since thunderstorms generate dozens of lightning strokes per second, the average volcanic lightning contribution for the global electric circuit is negligible at the global scale. However, for short periods of time, volcanic lightning provides interesting means for assessing transient response due to its localized nature. The highest number of strikes per square kilometer per year is about 200 in central Africa though such rate can be reached during a volcanic eruption in a matter of minutes. Although volcanic lightning is usually tenfold or more weaker than that due to thunderstorms, higher amounts of energy can be released in much shorter periods in the same location. Investigation of strong lightning strokes generated by volcanic eruptions is therefore useful for assessing local variations of the global electric circuit and propagation of low frequency electromagnetic waves. Remarkably, the most important contribution of volcanic eruptions for the global electric circuit is indirect. Ash plumes resulting from violent volcanic eruptions are dispersed in the stratosphere at regional or global scales, where ultrafine aerosol layers are formed, leading to atmospheric conductivity decreases by one order of magnitude or more (e.g., Tinsley, 2008). Effects of volcanic eruptions in electrostatic and electromagnetic noise have been investigated by Adler et al. (1999). 
Corona discharges are also observed during dust, sand turbulent lofting, such as $\sim 1 \mathrm{~m}$ long corona discharges observed during dry debris, gypsum sandstorms in New Mexico (Kamra, 1972a,b). Comparable scenarios involving dust devils and dust storms have been proposed for triggering corona discharging on Mars favored by the very dry Martian atmosphere. Saltation of sand particles and dust due to wind gusts induce impact charging near the surface, originating momentary sparks (Farrell et al. 1999). Massive dust storms, sometimes covering a significant part of the planet, would also be responsible for intense impact charging at much larger scales (Renno and Kok, 2008). Similar effects involving ice particles and cryovolcanism in much colder environments have been suggested (James et al., 2008). However, these scenarios for planetary atmospheric electricity have yet to be confirmed (Yair et al., 2008).

Anthropogenic Activity. In addition to natural processes mentioned above, anthropogenic-related lightning is also possible. Lightning has been triggered, deliberately or involuntarily, by rockets and aircraft. Thermonuclear explosions have been known to produce lightning, too. Laser technology has been used to force atmospheric breakdown in thunderstorms and induce lightning strokes. Nonetheless, the most common, ubiquitous artificial source of ELF and VLF waves are power line emissions at 50 or $60 \mathrm{~Hz}$ and their harmonics up to more than $3 \mathrm{kHz}$. Power line radiations can be employed to investigate wave-particle interaction (for a review, see Bullough, 1995). In addition to power line radiation, innumerable transmitters ranging from ELF to radar and microwaves are used worldwide. Although their contribution to electromagnetic sources global budget is marginal, a variety of transmitters can be used to locally monitoring or modifying ionospheric dynamics.

Lightning has been triggered by rockets carrying wire bobbins through thunderstorms. The wires unwind during rocket ascent to provide preferential high conductivity paths, where discharges are more likely to occur (e.g., Biagi et al., 2009). In some cases, aircraft flying within strong convective systems can trigger lightning (Mazur, 1989; Kito et al., 1995; Olsen et al., 2004; Jerauld et al., 2005). Statistically, each major aircraft is likely struck by lightning once a year and a well-known example is Apollo 12 being hit by lightning soon after takeoff (Rakov and Uman, 2007). During a thermonuclear surface burst, five discrete luminous channels were seen to start from the ground or sea surface at a distance of approximately $1 \mathrm{~km}$ from the burst point and to 
grow up into the clouds. The peak current of such lightning strokes was between 200 and $600 \mathrm{kA}$ (Uman et al., 1972; Colvin et al., 1987).

Another method of inducing lightning deliberately in thunderstorms is the utilization of ultra short laser pulses for producing laser-induced breakdown in the atmosphere (Ball, 1974; Khan et al., 2002). The laser pulse creates a channel of ionized gas through which the lightning stroke would be conducted to the ground. Under conditions of high electric field during two thunderstorms, Kasparian et al., 2008 observed a statistically significant number of electric events synchronized with laser pulses, at the location of the filaments. Laser-triggered lightning is intended to protect rocket launching pads, electric power facilities, airports, and other sensitive targets. Rocket- or laser-triggered events on demand would be also useful for studying ELF-VLF transient wave propagation because synchronization between the lightning stroke and receivers would be much more efficient.

\subsubsection{Sources Outside the Cavity}

Subsurface Phenomena. The connection between surges of electric and magnetic fields and seismic activity has been advocated for a very long time though unambiguous correlations are relatively recent (Gokhberg et al., 1982; Warwick et al., 1982). These observations provided new, effective means of investigating short-term precursors of earthquakes. The objective of this sub-section is to summarize possible effects associated with earthquakes and pre seismic activity that may impact boundary conditions, atmospheric electrical properties and represent an effective

electromagnetic source generation. Reviews about seismo-electromagnetic phenomena have been published by Johnston (1989, 1997), Parrot (1995), Molchanov and Hayakawa (1998). The two leading hypotheses concerning electromagnetic wave generation by earthquakes are direct wave production by rock compression near the focal point (piezoelectric and piezomagnetic effects) and electric discharges induced by redistribution of electric charges in the ground (electrokinetic phenomena). However, not all earthquakes produce measurable electromagnetic emissions.

Surface and subsurface resistivity variations have been noticed during pre seismic activity but conductivity changes of $\sim 0.1 \mathrm{mSm}^{-1}$ (Rikitake and Yamazaki, 1985; Utada et al., 1998) do not 
appreciably affect the cavity inner boundary. The magnetic field amplitude in the vicinity $(\sim 7$ $\mathrm{km}$ ) of the Loma Prieta earthquake $\left(\mathrm{M}_{\mathrm{s}} 6.0\right)$, for example, was $\sim 10 \mathrm{nTHz}^{-1 / 2}$, at $0.01 \mathrm{~Hz}$, several days before the initial shock (Fraser-Smith et al., 1990). A statistical analysis of several earthquakes in Japan showed that signal intensity increased by as much as $25 \mathrm{~dB} \mu$, at $81 \mathrm{kHz}$ (Gokhberg et al., 1982). Measurements of volcano-seismic activity in the Izu Island, Japan showed that ULF electric and magnetic fields were in the order of $10 \mu \mathrm{Vm}^{-1} \mathrm{~Hz}^{-1 / 2}$ and lower than $0.1 \mathrm{nT}$ before a few earthquakes and subsequent volcanic eruption (Uyeda et al., 2002).

In addition to in situ ground measurements, remote sensing techniques from low Earth orbit have been dedicated to earthquake research. Although correlation between pre seismic activity and ionospheric variability has been claimed, the subject remains controversial. A more reasonable result, however, is the observation of ionospheric disturbances related to gravity waves triggered by earthquakes (e.g., Tanaka et al., 1984; Wolcott et al., 1984). Incoherent scatter radar observations after earthquakes have shown large amplitude vertical velocity oscillations in the thermosphere. At 300-400 km altitude, velocities as high as $100 \mathrm{~ms}^{-1}$ have been measured (Kelley et al., 1985). Fast perturbations of the Earth crust can induce oscillatory motion in the atmosphere, generating gravity waves that propagate up to the ionosphere.

Although inducing electric and magnetic fields and modifying ground resistivity, earthquake contribution for the global electric circuit and ELF-VLF wave propagation is little. For example, surface conductivity differences due to land/sea dichotomy or precipitation (dry/wet regolith) prevail over ground resistivity variations triggered by earthquakes; additionally, average ELF electric fields are at least 50 times lower than those attributed to Schumann resonances and IAR signatures. Nevertheless, ELF-VLF transient response due to earthquakes deserves further investigation. Anomalous effects in Schumann resonance phenomena possibly associated with earthquakes have been claimed (Hayakawa et al., 2005, 2008). Even so, unambiguous causeeffect relation between Schumann resonance and earthquakes is difficult to establish because of the higher natural variability of lightning activity and of the ionosphere. The controversial earthquake lights phenomenon also deserves continued investigation, namely for assessing possible implications in the atmospheric electric circuit at a local scale. 
Solar and Interplanetary Contribution. Unlike the well defined, electrically stable, quasi-static surface, the outer boundary of the surface-ionosphere cavity is fuzzy, thick, irregular, and dynamic. Besides perturbations from below summarized in the previous sections, the dynamics of the outer boundary of the cavity is mostly driven by sources from above such as solar radiation, cosmic rays, energetic particle precipitation and, to a lesser extent, meteoroids. The role of cosmic rays in troposphere and stratosphere ionization is addressed in the following section. Solar radiation plays a global, permanent role in atmosphere ionization but its variations, mostly associated with the solar cycle, are only long term effects that are described in a number of monograph (e.g., Hunsucker and Hargreaves, 2002; Kelley, 2009). A review of ionospheric processes and dynamics can be found in (ISSI CPMPANION PAPER IN ionospheric electrodynamics and aeronomy).

Energetic particle precipitations include mainly solar proton events and electron precipitation in the auroral zones and from the radiation belts. The mesosphere and stratosphere close to the South Atlantic anomaly are also subject to a diffuse and weak energetic particle precipitation. Solar proton events enhance atmospheric ionization below $\sim 150 \mathrm{~km}$ altitude and have a major effect in the mesosphere and stratosphere at high latitudes where they can drastically modify the conductivity profiles and the EM wave propagation with the absorption of VLF waves increasing by $\sim 7 \mathrm{~dB}$ (Verronen et al., 2005). Roble et al. (1987) found significant Joule heating of the mesosphere and lower thermosphere during solar proton events. Reagan and Watt (1976) and Reagan et al. (1981) analyze satellite and radar measurements made during solar particle events and investigate direct effects in stratospheric ozone production. Solar proton events induce particle ionization in the mesosphere and upper stratosphere, enhancing the concentrations of short-lived $\mathrm{HO}_{\mathrm{x}}\left(\mathrm{H}, \mathrm{OH}, \mathrm{HO}_{2}\right)$ and long-lived $\mathrm{NO}_{\mathrm{x}}\left(\mathrm{N}, \mathrm{NO}, \mathrm{NO}_{2}\right)$ constituents. These molecules are then responsible for ozone depletion, creating a polar ozone cavity. After the event, ozone concentrations decreasing in the auroral region is $46 \%, 16 \%$, and $4 \%$ at altitudes of $49.5,41$, and $32 \mathrm{~km}$, respectively, reducing the total columnar ozone concentration by $\sim 2 \%$. Jackman et al. (2001) address the impact of solar flares in $\mathrm{HO}_{\mathrm{x}}$ and $\mathrm{NO}_{\mathrm{x}}$ concentration. Their measurements indicate short-term ( $\sim 1$ day) middle mesospheric ozone decreases of over $70 \%$ caused by shortlived $\mathrm{HO}_{\mathrm{x}}$ during the event with a longer-term ( $\sim 1$ week) upper stratospheric ozone depletion of up to $9 \%$ caused by longer-lived $\mathrm{NO}_{\mathrm{x}}$. Stiller et al. (2005) discuss the effects of $\mathrm{HNO}_{3}$ 
enhancements in stratosphere ion cluster chemistry due to $\mathrm{NO}_{\mathrm{x}}$ molecules produced in the mesosphere and continuously transported downward. McConnell and Jin (2008) further investigate the impact of solar variability in not only the mesosphere and stratosphere but also down to the troposphere.

The outer radiation belt consists mostly of electrons that are injected inward by radial diffusion from the geomagnetic tail following geomagnetic storms (Shprits and Thorne, 2004). Investigation of radiation belt processes is useful for lightning-related VLF wave propagation and for aeronomy studies, mainly in the mesosphere. Foster et al. (1986) discuss the connection of outer belt electron precipitation with ionospheric convection at high latitude and present a precipitation index that quantifies the intensity and spatial extent of precipitations as a function of the convection electric field. Callis et al. (1991) discuss the effects of relativistic electron precipitation in the D-region and long-term impact on stratospheric odd nitrogen levels. Results bear evidence for a strong connection between solar variability, the state of the magnetosphere, and the chemical climatological state of the middle and lower atmosphere. Newell and Meng (1992) and Newell et al. (1996) discuss the morphology of nighttime precipitation and introduce a detailed scheme for quantitatively classifying the radiation belt impact in the low ionosphere. Assessment of radiation belts particle precipitation is useful for not only aeronomy but also lightning-related wave propagation research. Imhof et al. (1983a,b) report on the precipitation of energetic electrons from the radiation belts by the controlled injection of VLF radio waves from the ground. Voss et al. (1984) present satellite measurements of electron precipitation by lightning; they find a one-to-one correlation between ground-based measurements of sferics and whistlers and precipitating energetic electrons detected onboard satellites. Burgess and Inan (1993) discuss the role of ducted whistlers in the precipitation loss of radiation belt electrons from the identification of ionospheric disturbances on subionospheric low frequency signals ('Trimpi events'). The Trimpi event is a phenomenon of amplitude or phase perturbation of subionospheric VLF signal propagation produced at the time of reception of a magnetospheric whistler, which scatters particles and induces D-region electron density enhancements (e.g., Carpenter et al., 1984). For electron energies of $\sim 100 \mathrm{keV}$, ducted whistlers may contribute as significantly as hiss to radiation belt equilibrium. Results also support the idea of ducted whistlers being responsible for burst electron precipitation. Rodger et al. (2003) address the 
significance of lightning-generated whistlers to the inner radiation belt radiation lifetimes. They estimate the contribution of whistler-induced electron precipitation for radiation belt loss rates, comparing such contribution with VLF transmitters and plasmaspheric hiss sources. Considering energetic electron precipitation fluxes driven by lightning rather than VLF wave perturbation observations, Rodger et al. $(2005,2007)$ provide regional and global estimates of energy deposition into the atmosphere, and study implications for ionization levels and neutral chemistry. They find that whistler-induced electron precipitation is never a significant source of ionization in the lower ionosphere for any location or altitude during the day. For nighttime, however, ionization in the low and high mesosphere is mainly due to galactic cosmic rays and whistler-induced electron precipitation, respectively. Horne et al. (2005) address the wave acceleration processes in the radiation belts. Characterization of the radiation belt particle precipitation in the low ionosphere is therefore useful for understanding ionization processes in the mesosphere.

The combination of solar ultraviolet radiation and energetic particles from the magnetosphere ionize the neutral constituents of the thermosphere, creating the ionosphere. Changes of solar wind related characteristics, namely velocity, shock waves, and high-speed streams are known to cause geomagnetic storms. Although the solar wind affects mostly the magnetosphere, perturbations can be observed down to the surface. For example, auroral optical phenomena are closely related to geomagnetic activity, solar proton events cause polar cap absorption in the lower ionosphere and increase electric conductivity in the high latitude stratosphere, and geomagnetic storms are known to cause power outage at mid and high latitudes. Rees (1995) reviews observations and modeling of ionospheric and thermospheric disturbances during major geomagnetic storms. On the other hand, Lastovicka (1996) investigates effects of geomagnetic storms in the lower ionosphere, middle atmosphere, and troposphere. Frahm et al. (1997) show that diffuse aurora is a significant source of ionization in the middle atmosphere because energetic electrons can cause excitation, dissociation, ionization, and heating of neutrals. Above and below $\sim 50 \mathrm{~km}$, respectively, most energy deposition, e.g., by ionization, results from direct electron impact and bremsstrahlung X-rays. In fact, in diffuse aurora and during geomagnetic storms, ionization from the bremsstrahlung component exceeds that due to the galactic cosmic ray background to altitudes as low as $30 \mathrm{~km}$. These effects usually enhance electric conductivity 
in the mesosphere and stratosphere, and increase radio wave absorption in the medium and high frequency range. ELF-ULF wave propagation is also influenced by solar and geomagnetic activities.

Radio waves play a dual role in the ionosphere because they can impact ionosphere dynamics directly and are also used for monitoring purposes, namely inferring medium propagation conditions. Radio waves have been used to induce ionospheric heating and plasma instabilities (e.g., Perkins and Kaw, 1971). Intense radio waves of frequency close to that of medium plasma frequency provide optimal conditions for inducing ionospheric plasma instabilities and, consequently, medium heating. ELF-VLF transmitters have been used for the same purpose and natural waves may induce similar effects, too. Dessler $(1959 \mathrm{a}, \mathrm{b})$ presents a first relevant description of ionospheric heating by MHD waves. Although contributing little during quiet periods, MHD waves may dominate ionospheric heating during magnetic storm activity. MHD waves also contribute to electron acceleration in the magnetosphere because, during major magnetic storms, enhanced fluxes of relativistic electrons in the inner magnetosphere have been observed to correlate with geomagnetic pulsations. Interaction between MHD waves and electrons leads to ionospheric heating because of particle precipitation and wave traveling through the ionosphere. Summers and Ma (2000) examine the mechanism of transit-time (bouncing) acceleration of electrons by low frequency fast magnetosonic MHD waves. They calculate the acceleration timescales of electrons due to interaction with MHD waves; for Pc4Pc5 pulsations (ULF range), seed electrons may reach the MeV energy range in a few hours. Thompson and Lysak (1996) address electron acceleration by MHD waves further. They discuss electron acceleration mechanisms involving Alfvén wave electric fields, namely via Landau and transit-time resonances. These waves can be partially trapped in the ionospheric waveguide and ionospheric Alfvén resonator. In addition to particle acceleration, Alfvén waves are a possible mechanism for generating electron conic distributions and particle precipitation in the ionosphere.

Space debris ablation in the atmosphere produces important transient effects, including visible emission, radio noise, and acoustic transients known as electrophonics. Meteor showers, for example, are known to be related to all these processes long time ago (Blagdon, 1784). 
Electrophonics, a phenomenon resembling to microphonics reversed effects, is the production of audible noise through direct conversion of very low frequency electromagnetic signals in to sound through transduction phenomena (Keay, 1980). It is worth mentioning that, sometimes, the acoustic signal on the ground precedes the corresponding optical flash observed during meteoroid ablation, which occurs in the D-region at altitudes of $80-120 \mathrm{~km}$, therefore suggesting generation of electromagnetic noise. Distinction between lightning and meteor shower transients is possible because the spectrum morphology is quite different; lightning and meteor spectra show a peak at about 5 and $0.5 \mathrm{kHz}$, respectively. Additionally, correlation between optical flashes and radio noise is significant (Price and Blum, 2000). Meteor phenomena and its detection techniques are reviewed in detail by Ceplecha et al. (1998), including radar and spectroscopic observations, trajectory geometry analysis, dynamics and ablation processes, and hazard assessment. In the context of this review, we shall briefly emphasize two major ideas: (i) meteor ablation delivers high concentration of metallic ions that locally modify the chemistry of the D-region during brief periods of time, and can be investigated with radar and spectroscopy techniques; (ii) meteor ablation produces ELF electromagnetic wave signatures, which can be combined with optical flash measurements to investigate meteoroid activity, mainly during meteor outbursts.

An intricate, interesting example connecting various layers of the Earth gaseous envelope is associated to wave-particle interaction. Particle precipitation from the radiation belts is among the most relevant phenomena to investigate the tropospheric-ionospheric coupling because whistler-induced particle precipitation in the mesosphere is related to lightning. Lightning produces whistlers that interact with cyclotron resonant radiation belt electrons, leading to particle acceleration and subsequent precipitation into the atmosphere. Particle precipitation then modifies medium conductivity, which affects wave propagation conditions.

Galactic Cosmic Rays. Cosmic rays are highly-energetic charged subatomic particles arriving from outer space, from solar, galactic, and extragalactic origin. Cosmic rays include protons ( $\sim 89 \%$ ), alpha particles ( $\sim 9 \%$ ), and heavier nuclei ( 1\%) spanning over a wide range of energies, from $\mathrm{MeV}$ to $\mathrm{EeV}$ and even higher; electrons constitute the remaining $1 \%$ of cosmic rays. Since their discovery about 100 years ago, cosmic rays have been studied from multiple, 
complementary perspectives dealing with astrophysics of compact objects, nucleosynthesis and star evolution, particle physics, radioisotope science, solar dynamics, interplanetary medium, magnetospheric processes, aeronomy, atmospheric electricity, radiation hazard to humans, and radiocarbon dating (e.g., Bieber et al., 2000; Grieder, 2001; Miroshnichenko, 2001; Schlickeiser, 2002; Stanev, 2009; Dorman, 2009). Here, we summarize the main implications for the surfaceionosphere cavity/waveguide, i.e., atmospheric electricity and aeronomy related processes.

Interaction between highly-energetic cosmic rays and the Earth gaseous envelope produces charged secondary cosmic ray components, which may involve particle multi-cascading of positron-electron pairs, protons, alpha particles, muons, photons, neutrinos, etc. Incident hadrons are subjected to strong interactions when colliding with atmospheric nuclei, mainly nitrogen and oxygen, producing nuclear spallation (Grieder, 2001). Since cosmic rays are electrically charged, high-energy particles interaction with the Earth environment results from not only atmospheric density variation with altitude but also geomagnetic field and solar wind dynamics. Consequently, cosmic ray flux shows an 11 year modulation due to the solar cycle, and for certain mechanisms also a 22 year periodicity. In addition to the variation with the solar cycle, cosmic rays intensity may drop suddenly by $15-30 \%$ due to sudden changes in solar activity (timescale of few minutes to several hours) followed by a gradual recovery (several hours to a few days) to the former intensity level; this phenomenon is known as Forbush decrease (Forbush, 1937, 1938, 1958) and is related to magnetic shock effects in the Earth magnetosphere due to coronal mass ejections. An opposite effect, where cosmic ray intensity increases up to 2-3 times the steady flux, is sometimes triggered by solar flares. Most galactic cosmic rays have energies too low to penetrate straight deep in the ionosphere and are channeled by the geomagnetic field toward the poles, inducing particle precipitation in the polar cap regions and contributing to aurora phenomena (Fisk et al., 1998). In addition to changes related to solar activity, a long term variation of high energy cosmic rays has been proposed to tentatively explain a 62 My periodicity in terrestrial fossil biodiversity. High energy cosmic rays also induced air showers ionize the atmosphere leading to changes in atmospheric chemistry and microphysical processes that can lead to cloud formation, including a low altitude cloud cover (e.g., Mellot et al., 2010). Similarly, Carslaw et al. (2002) and Kirkby (2007) discuss associations between climate trends and cosmic ray variations at a much shorter timescale. Observations suggest that cloud cover 
may be influenced by cosmic rays and two classes of microphysical mechanisms have been proposed to connect cosmic rays with clouds: an influence of cosmic rays on (i) the production of cloud condensation nuclei and (ii) directly on the global electrical circuit.

Since cosmic ray effects in the Earth environment are a function of atmospheric density and magnetic field, ionization rates show peculiar variations with altitude, magnetic latitude, longitude, azimuth, and the east-west direction. The cosmic ray relative intensity at high north latitudes is $50 \%$ larger compared to the equator and southern hemisphere because of geomagnetic cutoff imposed by the geomagnetic field to cosmic ray energies up to $\sim 15 \mathrm{GeV}$. Although smaller, there is a $15 \%$ longitudinal modulation in cosmic ray relative intensity $(+15 \%$ at $60^{\circ} \mathrm{W}$ and $-15 \%$ at $100^{\circ} \mathrm{E}$ ) introduced by the geomagnetic field asymmetry with respect to the equator and the South Atlantic anomaly. The geomagnetic field also drives an azimuthal response because of a cosmic ray zenith angle, $\theta$, intensity dependence with atmospheric depth $\left(\propto \cos ^{2}(\theta)\right)$ and a dipolar shape of the field. Additionally, because of geomagnetic field polarity and positive charge predominance in cosmic rays, an east-west asymmetry also develops. As a result of cross section increasing with atmospheric depth, the particle flux due to cosmic rays increases from the magnetosphere down to the stratosphere, reaching a maximum at a height of about $20 \mathrm{~km}$, which is called Pfotzer maximum (e.g., Pfotzer, 1936). Unlike ionospheric ionization due to solar radiation, this cosmic ray bombardment favors formation of atmospheric ions in the stratosphere (for a review, see Tinsley, 2008).

Cosmic rays contribute to ion-pair production down to the surface, and even deeper for the most energetic particles. Another important source of ionization is provided by ground radioactivity that results from alpha, beta, and gamma radiation. One particular source is radioactive radon and daughter products emanating from the ground. This ion-pair production rate has a 0.2 and 2$3 \mathrm{~km}$ scale height for ${ }^{220} \mathrm{Rn}$ and ${ }^{222} \mathrm{Rn}$, respectively. Radioactive gas releasing from the ground, namely during earthquakes, increases conductivity in the atmospheric boundary layer region by as much as a factor of $\sim 2$. Ionization rate from radioactive gases near the surface is up to $\sim 10 \mathrm{~cm}^{-}$ ${ }^{3} \mathrm{~s}^{-1}$ (Usoskin et al., 2009). 


\subsection{The AC and DC Global Electric Circuits}

The concept of a global electric circuit was first proposed by Wilson (e.g., Wilson 1906, 1920) to explain the existence of a vertical atmospheric electric field. The ground and ionosphere are usually regarded as equipotential surfaces and consequently defining the global electric circuit boundaries, but considering the ionosphere a perfect conductor is often too stringent. Since the ionosphere is dynamic and heterogeneous, establishing the altitude for an equipotential condition is not straightforward. The atmosphere, where the conductivity is much lower, is considered an imperfect dielectric, i.e., a medium with losses. The global electric circuit can be represented by a set of generators, resistors, and capacitors to simulate electric sources and medium behavior (e.g., Ogawa, 1985; Rycroft et al., 2000). Differentiation between AC and DC electric circuits response is determined from the relaxation time of the system, i.e., when either conduction or displacement current processes are dominant (Greifinger and Greifinger, 1978). The main characteristics of the global circuit include: (i) near-surface conductivity from $\sim 10^{-6} \mathrm{Sm}^{-1}$ for low contaminated ice and dry, poorly conducting rocks to $\sim 10^{-2} \mathrm{Sm}^{-1}$ for clay or wet limestone and $\sim 1 \mathrm{Sm}^{-1}$ for sea water; (ii) ionospheric conductivity $\sim 10^{-6} \mathrm{Sm}^{-1}$ in the D-region; (iii) vertical DC electric field varying between $\sim 100 \mathrm{Vm}^{-1}$ near the surface and a very small fraction of $1 \mathrm{Vm}^{-1}$ at $80 \mathrm{~km}$ (changing significantly with latitude and day-night dichotomy); (iv) upward currents predominantly originating from thunderstorms; (v) fair weather downward current density $\sim 1$ $\mathrm{pAm}^{-2}$; (vi) and a 200-300 kV potential difference between the ionosphere and the surface (e.g., Bering et al., 1998; Rycroft et al., 2008). Thunderstorm activity and associated phenomena drive a DC current of $\sim 1 \mathrm{kA}$, and each negative and positive lightning stroke carries an average AC current of $\sim 30$ and $\sim 300 \mathrm{kA}$ in the circuit, respectively, in less than $0.1 \mathrm{~ms}$. Singh et al. (2005) present an extensive, documented table with physical parameters of the global electric circuit.

Thunderstorms, namely those related to mesoscale convective systems, introduce significant perturbations to the global electric circuit. Strong convection produces charge separation in clouds and induces strong electric fields locally. The solar wind affects not only cloud microphysics, temperature, and dynamics in the troposphere but also the global electric circuit (e.g., Tinsley, 2000). In general, aerosol formation drives ion attachment to larger particles with subsequent decreasing of ion mobility and conductivity. Whenever an atmospheric electric field threshold is reached - air electrical breakdown is $\sim 15 \mathrm{kVcm}^{-1}$, depending of altitude and 
atmospheric conditions, but the actual threshold field is smaller due to mechanisms poorly understood - a lightning discharge occurs and broadband radiation is emitted (Rakov and Uman, 2007). Under such conditions, atmospheric conductivity varies significantly within the cloud and even above the thunderstorm (e.g., Holzworth et al., 1985). Local, intense electric fields in the order of $1-5 \mathrm{kVm}^{-1}$ were also observed during snow storms in Antarctica (Frank-Kamenetsky et al., 2010). Rycroft et al. (2007) argue that lightning activity contributes only $1 \%$ to maintain the high potential of the ionosphere. Specifically, moderate negative/positive lightning discharges would contribute to increase/decrease the ionospheric potential by $0.0013 \% / 0.014 \%$. Füllekrug (2004), analyzing intense positive lightning discharges, obtains similar results for $\sim 1$ min time scales. The total net upward current to the ionosphere due to lightning would be only $\sim 20 \mathrm{~A}$, contrasting with the $\sim 1000$ A contribution from conduction and convection currents associated to electrified shower clouds and charge separation within thunderclouds. The time scale for ionospheric potential recovery is $\sim 250 \mathrm{~s}$, similar to the relaxation time for the global circuit (Rycroft et al., 2000, 2008). Analyzing electric field measurements corresponding to wide time spans covering most of the twentieth century, Harrison (2002) found a secular declining of the atmospheric DC electric field. The $\sim 25 \%$ reduction of the electric field over most of that period is attributed to a decreasing of cosmic rays, which would increase the coupling resistance between the top of thunderstorms and the ionosphere. This secular variation would therefore imply modifications in global electric circuit and fair weather parameters, including ion concentrations and aerosol electrification. However, other parameters, namely surface temperature, also play a crucial role. For example, Price (2000) predicts that a $1 \%$ increase in global surface temperature may result in $\sim 20 \%$ increase in the ionospheric potential. The influence of cosmic rays on the global electric circuit and ramifications in climate variability in geologic timescales has been addressed from a perspective of cloud-aerosol processes (e.g., Kirkby, 2007). Cosmic rays may significantly influence the production of cloud condensation nuclei and, consequently, the electric circuit at a global scale, namely by modifying the downward current density. Harrison and Märcz (2007) analyze surface atmospheric electricity data at daily resolution to investigate heliospheric and cosmic ray effects on the global circuit and find annual, semi-annual, and 1.7 year periodicities in DC electric field magnitude. Interestingly, the latter is clearest during fair weather and reduced, often absent, when poor local weather conditions prevail. 
In addition to lightning and related tropospheric phenomena, the role of transient luminous events in the global electric circuit dynamics has been addressed concurrently with theoretical and experimental developments in sprites, blue jets, and elves generation mechanisms. Füllekrug and Rycroft (2006) assess the role of sprites to the global electric circuit and estimate that sprites individual contribution is lower than $44 \mathrm{mVm}^{-1}$, and Cummer et al. (2009) report a significant charge transfer of $-144 \mathrm{C}$ to the lower ionosphere in a gigantic jet event. Positive blue jets contribute to the charging of the global electric circuit, while negative gigantic jets discharge the circuit (Krehbiel et al., 2008). Pasko (2010) discusses effects of sprite and jet discharges on chemical processes and the global electric circuit. Detailed assessments of global electric circuit dynamics therefore require reliable data on generators, namely thunderstorm, lightning, and transient luminous events, and medium properties drivers such as cosmic rays and solar radiation.

Several reviews dedicated to the global electric circuit and connections to atmospheric electrification processes, cosmic ray and solar irradiance effects, cloud microphysics, and climate are available, offering detailed discussions of the subject (Harrison, 2004; Singh et al., 2004; Tinsley et al., 2007; Rycroft et al., 2008). Williams (2010) reviews origin and context of Wilson's pioneering ideas in atmospheric electrification and electron runaway in thunderclouds and a companion article by (ISSI COMPANION PAPER IN global electric circuit) provide a systematic analysis of the global electric circuit in troposphere-ionosphere coupling mechanisms.

\section{Modeling}

Accurate assessments of ULF-VLF wave propagation in the surface-ionosphere cavity/waveguide require utilization of appropriate formalism, boundary conditions, and medium parameterization. Several empirical models are available to derive medium properties, namely electron and ion density, collision frequency, and geomagnetic field distributions. While the MHD formalism is useful for describing the ionospheric waveguide, the ionospheric Alfvén resonator, and geomagnetic pulsations, Maxwell equations are necessary for solving ELF-VLF wave propagation in the cavity. Here we review analytical and numerical approaches to solve Maxwell and MHD equations and specific phenomenological models used for deriving medium 
dispersion relations. Characterization of electromagnetic sources or medium properties usually benefit from inverse problem techniques.

The most familiar, straightforward approximation for solving ELF wave propagation in the surface-ionosphere cavity considers an atmospheric conductivity profile with two scale heights, which is frequently known as the "knee-model". The scale height transition occurs when the displacement current is commensurate with the conduction current in the medium. Greifinger and Greifinger (1978) employ the knee-model approximation and cylindrical symmetry to determine the electromagnetic field distribution and eigenfrequencies of the cavity. Sentman (1990) utilizes a similar approach employing spherical symmetry for eigenmode characterization. Mushtak and Williams (2002) extend the knee-model approach to determine modal frequencies, wave phase velocities, and wave attenuation with improved accuracy. Nickolaenko and Hayakawa (2002) review these and asymmetric approximations of the cavity as well. Analytical approximations provide valuable results for assessing propagation and resonance mechanisms but are insufficient for obtaining accurate estimates, particularly if daynight asymmetry or polar heterogeneity is incorporated in the model. A similar situation occurs in planetary environments because thick cavities imply strong coupling between the longitudinal and transverse modes (Simões et al., 2007) and because refraction phenomena occur in the low atmosphere (Simões et al., 2008b), reducing model accuracy.

Modeling of ionospheric waveguide (magnetosonic mode) and ionospheric Alfvén resonator (Shear Alfvén mode) implies solving MHD equations with specific parameterization. Although the magnetosonic and Shear Alfvén modes are often modeled separately, coupling between modes is sometimes important. Consistent analytical approaches are available for modeling the ionospheric waveguide and ionospheric Alfvén resonator. Greifinger and Greifinger (1968) address the theory of MHD wave propagation in the ionospheric waveguide, the relevance for investigating geomagnetic pulsations, namely "pearls", and implications for ionospheric ducting phenomena about the Alfvén velocity minimum near the F-peak. Polyakov and Rapoport (1981) discuss a similar theory for wave propagation in the Alfvén shear mode (i.e., transverse wave propagation in ionospheric ducts), as opposed to the magnetosonic mode which is connected to ionospheric ducting. Far from the poles, the ionospheric waveguide and ionospheric Alfvén 
resonator correspond approximately to horizontal and vertical wave propagation in the ionosphere, respectively. When losses are taken into account, Hall conductivity couples the propagation modes and a more general formulation is employed (Lysak, 1999).

Comparable formulations are used for investigating geomagnetic pulsations of lower frequency, whistler-mode propagation, and wave-particle interaction related to radiation belt electron precipitation. Carpenter and Anderson (1992) discuss an empirical model of equatorial electron density derived from satellite-based whistler signatures. The model is specially suited for investigating whistler-mode propagation, lightning, and radiation belt particles from whistlermode dispersion measurements. Yagova et al. (1999) study diurnal variations of the magnetospheric Alfvén resonator parameters to characterize mid latitude Pc 3-4 pulsations. Bortnik et al. (2006) present a wave-particle interaction model to assess temporal signatures of radiation belt particle precipitation induced by lightning-generated whistlers reflected in the magnetosphere. Although not strictly matching the scope of the present work, these models are useful for understanding the coupling between the ionosphere and the magnetosphere because the troposphere-ionosphere coupling is also driven from above, namely in the auroral region where particle precipitation happens.

A variety of time and frequency domain techniques is available to solve MHD and Maxwell equations, namely Finite Difference Time Domain (FDTD), Finite Difference Frequency Domain (FDFD), Finite Element (FE), and Transmission Line Matrix (TLM) analysis. Most techniques can be used for eigenvalue, harmonic propagation, and transient problems. While TLM provides the simplest approach, the FE technique offers robust solutions for intricate geometries. Simpson and Taflove (2007) present a review of progress in FDTD modeling of subionospheric propagation, focusing in ELF-VLF wave propagation related to lightning sources and radiation, remote sensing of localized ionospheric anomalies, and Schumann resonances. Cummer (1997) discusses FDTD methods specially suited for solving wave propagation in isotropic cold plasma and Young (1994) compares both FDTD and FDFD techniques aiming a similar purpose. For modeling field interaction and propagation in anisotropic media with generalized permittivity and permeability tensor formulation, Rappaport and McCartin (1991) discuss FDFD using unstructured mesh methods to solve ionospheric scattering problems. 
Simões et al. (2007, 2008b, 2008c) discuss FE methods applied to ELF-VLF wave propagation in the surface-ionosphere cavity/waveguide, namely for solving eigenvalue, harmonic propagation, and transient problems related to lightning morphology and distribution, sferics, and Schumann resonances. Christopoulos (1995) describes the TLM model in detail to solve 3D wave propagation problems, emphasizing analogies between network circuits and physical systems; distributed circuits are used to define propagation conditions and medium properties. Although FE modeling can be applied to more intricate domains than finite difference, which is more robust than transmission line methods, the TLM analysis is faster and sometimes chosen when processing time and memory constraints are crucial.

The characterization of the medium from field measurements, i.e., by solving the inverse problem, is invaluable both for troposphere and ionosphere modeling. The inverse problem can be also used for source description, namely lightning distribution in the cavity. Whereas seeking for inverse problem analytical solutions is commonly impracticable, numerical modeling may provide meaningful results. He et al. (1996) show an optimization approach to solve a 1D electromagnetic inverse problem for reconstructing medium characteristics of stratified dispersive and dissipative slabs. Gustafsson and He (2000) present a similar approach to solve 2D electromagnetic inverse problems and simultaneous reconstruction of the permittivity, permeability, and conductivity of the medium by means of the iterative conjugate gradient algorithm. To investigate mode conversion in weakly magnetized plasma, Yin and AshourAbdalla (1999) describe the wave energy coupling and mode structure of electrostatic Langmuir and upper-hybrid branches, and electromagnetic ordinary, extraordinary, and Z modes. Sharapov et al. (2001) apply the inverse problem approach to solve MHD equations of toroidal Alfvén eigenmodes in tokamaks; similar techniques can be applied to toroidal geomagnetic pulsations (Pc 5 waves) and a modified approach is useful for inferring the ion density profile from ionospheric Alfvén resonator eigenfrequency measurements. Miura (1999) proposes an MHD inverse problem to investigate phenomena related to the magnetopause Kelvin-Helmholtz instability. Ando et al. (2005) employ finite difference analyses of Schumann resonance data for lightning distribution reconstruction. Although finding reliable, accurate solutions is not straightforward, inverse problem techniques can successfully be applied to investigate source and medium characteristics from electromagnetic field measurements. 
Consistent solutions of wave propagation problems in the surface-ionosphere cavity/waveguide require accurate medium parameterization. The geomagnetic field of the Earth environment can be computed from the International Geomagnetic Reference Field (IGRF). The IGRF model consists of a spherical harmonics expansion of the geomagnetic potential, whose coefficients are determined empirically (Finlay et al., 2010). The model provides the 3D geomagnetic field distribution from the core to the magnetosphere, including the secular variation rate. Another option to compute the geomagnetic field is utilizing the POtsdam Magnetic Model of the Earth (POMME), which also evaluates the magnetic field of the ionosphere and magnetosphere (Maus et al., 2006).

The collision frequencies between electrons, ions, and neutrals can be calculated from the International Standard Atmosphere (ISA), which provides the thermodynamic properties of the atmosphere, namely pressure, temperature, and density as function of altitude (Lide et al., 2010). A more elaborate approach is offered by the Naval Research Laboratory Mass Spectrometer Incoherent Scatter radar (NRLMSIS-E-00) empirical model that provides the composition, temperature, and density distribution of neutrals from ground to the thermosphere (Picone et al., 2002).

There are three main classes of models that can be used to calculate the electron density and ion composition of the ionosphere: empirical, physics-based, and assimilative. The International Reference Ionosphere (IRI) empirical model (Bilitza, 2001, 2009) is the international standard for computing both electron and ion 3D distributions in the ionosphere, including electron density and temperature, and ion density, temperature, and composition. The model may also be used to provide estimates of equatorial vertical ion drift, total electron content, F-peak density, and spread-F probability. For given location, time, and date, IRI provides monthly averages of electron and ion parameterization in the altitude range from 60 to $2000 \mathrm{~km}$.

There are several options for physics-based models. The US Naval Research Laboratory has developed SAMI (Sami is Another Model of the Ionosphere) under 2D and 3D formulations (SAMI2 and SAMI3, respectively). The SAMI3 code provides field line integrated conductance 
and is especially suited for global circulation models, namely computing DC electric fields and ion drifts at low- and mid-latitude. SAMI3 evaluates the plasma dynamics and chemistry of seven ion species in the altitude range $80-20,000 \mathrm{~km}$. The temperatures of the electron population and each of component ion species are computed; the total electron content is also available. In addition, the $\mathbf{E} \times \mathbf{B}$ drift motion of the plasma is included for zonal and meridional electric fields, where $\mathbf{E}$ and $\mathbf{B}$ are the DC electric and magnetic fields (Huba et al., 2000, 2005; Krall et al., 2009). The Coupled Thermosphere-Ionosphere Plasmasphere Electrodynamics model (CTIPe) consists of four distinct component models that are fully coupled in energy, momentum, and continuity (Millward et al., 2001). The four components include a global thermosphere model, a high-latitude model, an ionosphere/plasmasphere model for the mid to low latitudes, and a model of the electrodynamic coupling between the ionosphere and thermosphere at low latitudes. CTIPe provides ion and electron densities from $140-2000 \mathrm{~km}$, as well as the height and density of the F2 peak. The US Air Force Research Laboratory has been developing a Parameterized Ionospheric Model (PIM) that is capable of generating global electron number density as well as maps of total electron content. The PIM code also provides the heights of the E, F1, and F2 peaks and their respective plasma frequencies as a function of geophysical parameterization, namely solar and geomagnetic activity indices (Daniell et al., 1995; Retterer et al., 2005). The Utah State University Global Assimilation of Ionospheric Measurements (USU-GAIM) is a physics-based ionosphere-plasmasphere-polar wind model with a Kalman filter to assimilate real-time electron density measurements from a variety of ground-based and in situ platforms, including GPS, digisondes, and the DMSP satellites (Schunk et al., 2004).

Investigation of troposphere-ionosphere coupling mechanisms requires a fundamental comprehension of not only what processes take place in the Earth's gaseous envelope but how the properties of the medium are affected by such processes. Therefore, theoretical and phenomenological models, analytical and numerical approaches, direct and inverse problem solutions contribute to a better understanding of the Earth's environment because physical processes and the response of the medium are frequently interrelated. 


\section{Discussion}

Alfvén Waves. The ionospheric magnetosonic waveguide and the ionospheric Alfvén resonator contribute to investigating connections among the troposphere, mesosphere, and thermosphere from their ULF wave signatures, namely geomagnetic pulsations. Specifically, for ionospheric Alfvén resonator studies, association between tropospheric and ionospheric phenomena is dual because of implications for wave propagation and electromagnetic source classification. First, we discuss relevance for medium characterization. Lysak $(1999,2004)$ investigates the dependence of Alfvén wave propagation on ionospheric parameters, namely the Pedersen and Hall conductivities. Ionospheric Alfvén resonator ground signatures are strongest when the Hall conductivity is greater than the Pedersen conductivity. The fundamental IAR mode is found not to couple to the magnetosonic mode, while higher harmonics of the wave are readily ducted through the ionospheric waveguide. In addition to the troposphere-ionosphere connection, investigation of ionospheric Alfvén resonator also contributes to an understanding of the magnetosphere-ionosphere coupling, because the resonator top boundary is sometimes located several thousand kilometers above the F-peak. Besides, this approach is useful to modeling ducted Pc1 oscillations that can propagate thousands of kilometers in the ionosphere. Surkov et al. $(2005,2006)$ investigate the ionospheric Alfvén resonator excitation mechanism due to nearby thunderstorms, and suggest lightning is the major source driving the resonator. Sharp impulses in dynamic spectrograms recorded at high magnetic latitude are in a one-to-one correspondence with ionospheric Alfvén resonator signatures, suggesting nearby lightning discharges are followed by transient excitation of the resonator. Additionally, they also claim that the contribution from nearby thunderstorms prevails over remote, e.g., tropical, thunderstorm activity. This result is somewhat expected due to the local nature of the ionospheric Alfvén resonator; lightning distribution is expected to have a more global impact in the magnetosonic mode because of ducted propagation between the northern and southern hemispheres. Demekhov et al. (2000) discuss the connection between the ionospheric Alfvén resonator and Pc1 waves, namely the role of geomagnetic pulsations in resonator excitation. Understanding under what conditions Pc1 waves are a cause or a consequence of the ionospheric Alfvén resonator action would present invaluable information for troposphere-ionosphere- 
magnetosphere coupling research. The ionospheric Alfvén resonator can therefore act as a filter for unstructured large band electromagnetic noise produced by lightning or as a coupled oscillator for geomagnetic pulsations. A combination of several scenarios is possible: (i) lightning may pump IAR modes directly; (ii) transient luminous events may excite the resonator; (iii) magnetospheric Pcl waves may inject energy in the resonator; (iv) in situ ionospheric or magnetospheric processes related to the Kelvin-Helmholtz instability may produce medium turbulence and induce wave filtration in the resonator; (v) whistler-induced electron precipitation may generate electrostatic and electromagnetic noise in the lower boundary of the resonator. Either way, resonator characterization would be valuable for estimating ionospheric electron and ion density profiles. Classification of multiple eigenfrequencies and Q-factors of the resonator provides fundamental inputs for solving the inverse problem and eventually inferring density profiles that best fit spectra.

ELF Waves and Schumann Resonance. Global, continuous monitoring of ELF waves has been used for addressing phenomena related to not only lightning distribution but also low ionosphere variability. Some of the most challenging issues are related to quantifying regional contributions to the total lightning budget. Since lightning is closely related to thunderstorm electrification processes, monitoring Schumann resonance peaks variability - amplitude, frequency, and Qfactor - contributes to investigating atmospheric electricity at a global scale, including possible connections to climate trends. Identification of correlations between ELF spectra, and lightning activity as well, and atmospheric thermodynamic parameters is valuable for understanding possible links between hydrodynamic and electrodynamic processes. Although extremely demanding, solving the ELF inverse problem to characterize electromagnetic sources from Schumann resonance spectra provides important means for trends unambiguous identification between lightning and atmospheric parameters such as temperature and water vapor. A similar concept can be applied to investigate connections between atmospheric electricity and low ionosphere variability related to plasma depletions, scintillations, magnetospheric phenomena, and solar activity.

Schumann Resonance and Ionospheric Variability. Unambiguous detection of Schumann resonance signatures onboard the C/NOFS satellite, in the altitude range $400-850 \mathrm{~km}$ at 
equatorial latitudes, offers new means for investigating ELF wave propagation in the ionosphere (Simões et al., 2011). Although a background noise increasing is frequently observed at night and Schumann resonances are sometimes buried in the noise, nighttime measurements offer better propagation conditions because of significantly lower wave attenuation in the ionosphere compared to daytime. Besides, significant correlation between Schumann resonance amplitude and lightning activity is plausible. In general, Schumann resonance amplitude increases when the satellite flies over regions with enhanced lightning rate, corroborating the connection among Schumann resonance amplitude, lightning, and thunderstorm activity. ELF-flashes and ELFbursts (Q-bursts) are detected from orbit. C/NOFS measurements also show that Schumann resonance electric field is approximately perpendicular to the geomagnetic field. Although propagation in the whistler and extraordinary modes are perhaps compatible with C/NOFS observations, further developments are necessary to understand the leakage mechanism comprehensively. The leakage mechanism that allows ELF waves to escape the surfaceionosphere cavity is not understood thoroughly because analytical and numerical models currently available consider Schumann resonance modes confined to the cavity, i.e., between the surface and $\sim 100 \mathrm{~km}$. It is nevertheless expected that modeling and ongoing C/NOFS measurements contribute to consolidate present knowledge of ELF wave propagation in the ionosphere. Schumann resonance signatures remote detection provides invaluable information for atmospheric electricity multidisciplinary analyses: (i) ELF measurements can be combined with lightning optical detectors for nighttime operations; (ii) ELF measurements can be combined with optical cameras and spectrometers aiming thunderstorm electrification process studies; (iii) satellite measurements are complementary to ground stations because electric fields are partially decoupled in the ionosphere due to anisotropy, providing additional constraints to solve wave propagation inverse problems, i.e., characterizing electromagnetic sources from fields distribution; (iv) remote, concurrent ELF and DC field measurements contribute to the investigation of the global electric circuit, namely parameterization of energy budget and processes relaxation time; and (v) addressing ionospheric variability taking into account perturbations both from below (surface, troposphere, and stratosphere) and above (magnetosphere and beyond). Indeed, characterization of ELF wave propagation through the ionosphere contributes to not only an understanding of the local plasma dynamics but an assessment of the electric environment below. Moreover, Schumann resonance measurements 
from orbit offer supplementary means for troposphere-ionosphere coupling mechanisms research. Future Earth observation missions may explore new remote sensing capabilities employing ELF measurements for addressing atmospheric electricity and its connections to weather.

Ionospheric Irregularities and Dynamics. It is well established that not only lightning produces shock waves audible as thunder but thunderstorms can trigger hydrodynamic waves. Atmospheric gravity waves can be generated by various surface and tropospheric phenomena, namely weather related - thunderstorms, hurricanes, tornadoes - impulsive auroral zone momentum injection, earthquakes, tsunamis, and volcanic eruptions, whose effects are sometimes identified in the ionosphere. For example, Taylor and Hapgood (1988) identify a thunderstorm as a source of short period gravity waves in the upper atmospheric nightglow emissions, and Kelley et al. (1985) report large-amplitude thermospheric oscillations induced by an earthquake. It is not certain, however, whether lightning or transient luminous events could trigger strong hydrodynamic effects in the mesosphere or thermosphere. On the one hand, lightning is usually associated to thunderstorms, which induce atmospheric strong convection that prevails over lightning shock wave disturbances. On the other hand, establishing causality between lightning acoustic and electromagnetic transients far from the source is not straightforward. Although connections between thermospheric gravity waves and lightning have been suggested (e.g., Chiu et al., 1979; Liao et al., 1989; Kaladze et al., 2008), a positive correlation is possibly due to deep convective activity in thunderstorms. Davis and Johnson (2005) identify a lightning-induced intensification of sporadic-E. Because no ionospheric response to low-pressure systems without lightning is detected, they conclude that sporadic-E localized intensification can be attributed to lightning. According to them, medium density enhancement could be explained by hydrodynamic or electromagnetic processes, i.e., vertically propagating gravity waves or vertical electrical discharge, respectively. Various electromagnetic effects of lightning in the mesosphere, thermosphere, and beyond are well documented, namely mesospheric heating and whistler-induced particle precipitation, but hydrodynamic effects triggered by lightning are more difficult to assess. Nevertheless, a phenomenon known as explosive spread-F deserves to be discussed in detail. Spread-F is a term used to identify large ionospheric plasma depletions often observed in the equatorial regions during nighttime; spread- 
$\mathrm{F}$ is related to hydrodynamic phenomena started by the Rayleigh-Taylor instability at the bottom side of the ionospheric F-peak (Kelley, 2009). Whilst conducting equatorial backscatter measurements at the Jicamarca Radio Observatory in Peru, Woodman and La Hoz (1976) and Woodman and Kudeki (1984) observed a sudden, sharp increasing in spread-F signatures sometimes accompanied by transients in a high frequency receiver. They noticed radar echo measurements attributed to spread-F occurring simultaneously with transients in a receiver. The transients in the receiver were attributed to lightning strokes. Because of morphological structure, those bursts of backscattered radar are termed explosive spread-F and are associated to plasma irregularities at altitudes near $250 \mathrm{~km}$. Woodman and Kudeki (1984) and Kelley et al. (1984) suggest a causal relationship between lightning and explosive spread-F since lightningproduced transient electric fields can penetrate in the ionosphere, drive the instability mechanism, and trigger spread-F formation locally. The latter study proposes that transient electric fields cause an immediate $\mathbf{E x B}$ drift of electrons while ions remain mostly unaffected, where $\mathbf{E}$ and $\mathbf{B}$ are the lightning-induced electric field and the background magnetic field, respectively. The previous assumption is valid when the time constant of the transient is longer than the electron gyroperiod but shorter than the ion gyroperiod. The resulting differential motion generates a current that may induce medium instabilities. Because troposphericionospheric coupling assessments are useful for multidisciplinary analyses, namely medium instabilities seeding and irregularities generation, measurements combining hydrodynamic and electromagnetic techniques would be valuable for validating acoustic and electromagnetic connections between lightning and spread-F, and improving our understanding of the interaction mechanisms.

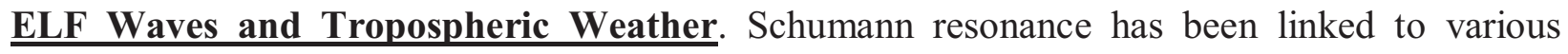
tropospheric and ionospheric phenomena related to the electromagnetic source and medium properties. Thunderstorm effects in ELF wave propagation conditions are especially important because of its connection to tropospheric water vapor, ice distribution in the tropopause, lightning, and aerosols. Williams (1992) claims an association between Schumann resonance and temperature fluctuations in the tropical regions because of a correlation between lightning flash rate and thermodynamic properties of the atmosphere. Price (2000) discusses evidence for a link between global lightning activity and upper tropospheric water vapor. Tropospheric water vapor 
plays an important role in climate, including a direct impact in tropospheric chemistry, namely aerosol formation, cloud albedo, and as a greenhouse gas. The latter suggests that water vapor changes in the upper troposphere can be inferred from records of global lightning activity, because deep convective thunderstorms transport important amounts of water into the upper troposphere. In some case, water ice can be transported into the tropopause. Since lightning induces Schumann resonances, ELF wave monitoring would contribute to a better understanding of water ice transport into the tropopause and trends in climate variability. These principles are indeed valuable for strengthening the link between thermodynamic and electromagnetic effects in the atmosphere. In particular, they are useful for assessing the contribution of the three major tropical thunderstorm centers to global lightning budget and ELF wave propagation. Therefore, a clear identification of these effects in ELF spectra, namely Schumann resonance signatures, would offer an independent approach to address trends in climate change.

Atmospheric Electricity and Tropospheric Weather. Weather fronts, hurricanes, and tornadoes are known to influence tropospheric hydrodynamics and ionospheric electrodynamics. Gravity waves, for example, have been associated to spread-F, medium-scale traveling ionospheric disturbances, and sporadic-E and are important to investigate the coupling between multiple layers, from the troposphere to the thermosphere (ISSI COMPANION PAPER IN aeronomy ionospheric electrodynamics). Tropospheric phenomena, mainly thunderstorms, also drive electrodynamic processes such as charge separation, lightning, and transient luminous events. Because ELF-VLF wave signatures of the surface-ionosphere cavity are closely related to lightning activity, investigation of Schumann resonance, sferics, and whistlers contributes to address lightning variability. ELF-VLF electric and magnetic field measurements from multiple stations can be used to solve the inverse problem and estimate electromagnetic sources distribution in the cavity. Specifically, Schumann resonance Q-bursts may be valuable for assessing specific features related to hurricanes and tornadoes. In addition to the correlation between lightning and thunderstorm activity at a global scale (e.g., Rakov and Uman, 2007), investigation of local connections between lightning and hurricanes and tornadoes is useful. Cecil et al. (2002) investigate lightning characteristics of hurricane eyewalls and rainbands and find that the outer rainband region produces more lightning, a similar feature also reported by Khain et al. (2008), claiming that intense and persistent lightning takes place within a 250-300 
$\mathrm{km}$ radius ring around the hurricane center. While investigating tornadoes and hailstorms, MacGorman and Burgess (1994) conclude that positive ground flashes can dominate in some severe storms for periods ranging from $30 \mathrm{~min}$ to several hours despite negative ground flashes usually dominate cloud-to-ground lightning activity. Additionally, they found a significant association between strong positive cloud-to-ground lightning and severe storms that involve hail or tornadoes. In the vicinity of severe storm, ELF Q-burst enhancement is expected. A similar conclusion might be drawn for sferics and whistlers, too. ELF-VLF spectral variability could therefore contribute to a better electrical characterization of severe thunderstorms.

Investigation of VLF wave propagation related to natural and anthropogenic sources has significantly contributed to an understanding of low ionosphere dynamics. Barr et al. (2000) review the subject thoroughly and Bosinger and Shalimov (2008) analyze lightning signatures combining the VLF and ULF ranges, discussing similarities and differences among Trimpi effect, ionospheric Alfvén resonator, and geomagnetic pulsations. Although subionospheric wave propagation related to VLF transmitters and whistlers present the most obvious link to study the troposphere-ionosphere coupling, other phenomena also contribute to monitoring ionospheric dynamics and the impact of tropospheric processes in the layers above. Sferics and tweeks offer additional means to investigate subionospheric wave propagation in the surface-ionosphere waveguide. Unlike VLF transmitter characteristics such as power and polarization that are adjustable and possibly synchronized with receivers, sferics can be used to investigate not only ionosphere reflection properties but also lightning characteristics. In addition to whistler-mode wave propagation and whistler-induced electron precipitation, whistler-related electrostatic waves and plasma turbulence have been studied. Baker et al. (2000) reported the generation of electrostatic emissions by lightning-induced whistlers above thunderstorms using sounding rockets that overflew active storm cells. Electric field measurements and lower hybrid resonance frequency show similar features as function of altitude, suggesting electrostatic emissions may be responsible for ionospheric irregularities that cause explosive spread-F. Berthelier et al. (2008) discuss lightning-induced plasma turbulence and ion heating in equatorial ionospheric plasma depletions. They present observations of equatorial plasma waves that show evidence for lower-hybrid solitary structures and the simultaneous occurrence of ion heating in deep, largescale equatorial plasma depletions that form at night during disturbed geomagnetic conditions. 
These phenomena are associated to lightning-induced whistlers and bear evidence for plasma turbulence and electrostatic waves playing a role in troposphere-ionosphere coupling. Along with whistler-related phenomena, a connection between subionospheric VLF signal perturbations and seismic activity has also been addressed. Molchanov and Hayakawa (1998) suggest that some subionospheric VLF signal perturbations are possibly related to earthquakes; a transient oscillation with a period of 5-10 days observed several days before a strong earthquake and lasting days or weeks after the event is reported. They claim that long-period gravity waves related to earthquakes could possibly explain the observations. Nevertheless, theories have not been able to explain the mechanism yet, namely establishing the causality principle for those observations. Significant effort has been made to understand such kind of surface-ionosphere coupling though further comprehensive measurements are necessary to address the problem thoroughly.

Surface Phenomena. Connections between hydrodynamic, electrostatic, and electromagnetic processes and earthquakes have been unequivocally established. The circumstances are nonetheless different concerning preseismic activity since establishing precursor causal nexus is rather difficult. In particular, there are several subjects where the impact of earthquakes deserves further attention, namely atmospheric conductivity, geomagnetic pulsations, whistlers, discharging phenomena, and atmospheric chemistry whenever volcanic eruptions are involved. Atmospheric conductivity enhancements within the atmospheric boundary layer have been monitored from radon content variability near the surface. However, the nature of earthquake lights phenomenon remains unclear and its possible connection to atmospheric conductivity enhancement uncertain. Establishing whether the phenomenon is intrinsically linked to earthquake activity would be a first step. Then, identifying key role processes involving conductivity, electric field, and ionization variability would be invaluable. A correlation between ELF-VLF waves and earthquakes has been addressed by the seismology community. Employing statistical analyses, Hayakawa et al. (1993, 2005, 2008) suggest anomalous effects in Schumann resonance spectra might be associated to earthquakes. They also claim that seismic activity modifies the rate and intensity of whistlers. However, it is not evident what kind of mechanisms would be implicated. Earthquakes could amplify ELF-VLF wave sources and their electrostatic and electromagnetic processes. More likely though, earthquakes generate gravity waves that 
possibly modify the upper boundary of the cavity, affecting ELF wave propagation, or inject momentum in the thermosphere, disturbing whistler propagation in the ionosphere. More elaborated studies are nevertheless necessary to first corroborate previous results and then identify and characterize the mechanisms involved. For example, studying subionospheric VLF radio signals and local, medium-scale traveling ionospheric disturbances could be useful in the context of seismic activity. Investigation of major volcanic eruptions to characterize their impact in atmospheric conductivity modification, local lightning budget, and low ionosphere disturbances induced by gravity waves would be valuable, too.

\section{Summary}

The investigation of troposphere-ionosphere coupling mechanisms is quite challenging because it requires an understanding of a wide variety of atmospheric science branches, from meteorology to aerology, from tropospheric weather to space weather. For this reason, individual studies can often seem segmented and disjointed when compared to other fields of research. One of the most remarkable examples of a specific topic requiring integrated, coherent studies is that of lightning. The study of this seemingly simple topic testifies as to how atmospheric electricity, specifically fulminology, is interconnected to meteorology, atmospheric dynamics and chemistry, aeronomy, space plasma physics, and both hydrodynamic and electromagnetic wave propagation. Ramifications to climatology may also be implied in a broader sense. Lightning is an atmospheric discharge accompanied by thunder and usually occurring associated to thunderstorm electrification processes during deep convection. Although the mechanism is not understood thoroughly - the most widely accepted theories involve electron runaway breakdown initiated by cosmic rays or positive streamers triggered by hydrometeors - electric fields can build up until the atmospheric breakdown is reached and a discharge happens. Most discharges occur within the clouds but an electric charge can also be transferred between the cloud and the ground or the lower boundary of the ionosphere. The discharge ionizes the medium, modifying the chemistry locally - ozone increasing is a good example - and produces acoustic and electromagnetic waves. The lightning stroke radiates in a wide frequency band, showing a peak in the VLF range. Lightning is associated with several wave propagation phenomena, including electromagnetic oscillations in the ELF range (Schumann resonance), and transients in the VLF (sferics, tweeks, and whistlers) and possibly ULF (IAR signatures and geomagnetic pulsations). In the case of 
whistlers, lightning-induced particle precipitation in the mesosphere can occur, therefore modifying wave propagation conditions in the low ionosphere because plasma heating and ionization are often enhanced locally. This mechanism also demonstrates the implications of atmospheric electricity for wave-particle interaction; moreover, the significance of lightning for the DC global electric field is unquestionable. In a broader sense, due to a close connection to thunderstorms, lightning is a useful tool for weather monitoring. Low frequency electromagnetic wave propagation, being closely related to lightning, can be used for identifying and characterizing weather patterns and, hypothetically, climate trends. The diagram shown in Figure 2 summarizes the most relevant sources and processes for investigation troposphere-ionosphere coupling processes.

Investigation of ULF-VLF wave propagation contributes to our understanding of the Earth atmospheric environment in a broader context. Some of the most challenging issues, possibly entangled with troposphere-ionosphere coupling mechanisms, are summarized as follows:

- Determining to what extent lightning activity contributes to pump the ionospheric Alfvén resonator;

- Establishing whether some geomagnetic pulsations, mainly Pc1, are a cause or an effect of the ionospheric Alfvén resonator;

- Solving the ionospheric Alfvén resonator inverse problem to derive electron and ion density profiles variability from ULF spectra;

- Establishing an indisputable connection between Schumann resonance spectra and thermodynamic parameters, namely tropospheric water vapor and temperature;

- Solving the Schumann resonance inverse problem to investigate lightning distribution and variability from ELF spectra;

- Identifying possible correlations between long term Schumann resonance variability and climate trends;

- Investigating ionospheric variability effects in Schumann resonance spectra, namely during the recent solar minimum;

- Understanding the surface-ionosphere cavity leakage mechanism for ELF waves;

- Accurate quantification of conductivity/ionization variability effects in ULF-VLF wave propagation, namely from galactic cosmic ray, particle precipitation, meteoroids, volcanic activity, and pollutants; 
- Monitoring transient wave propagation to investigate possible correlations between atmospheric discharge processes, namely lightning and transient luminous events, and VLF waves;

- Validating the connection between explosive spread-F and lightning, and quantifying the contribution of electrodynamic processes to spread-F global budget;

- Confirming the role of earthquakes in Schumann resonance and whistler transient enhancement and geomagnetic pulsations generation;

- Verifying earthquake contribution to stratospheric (and above) conductivity enhancements.

Finally, the recent, peculiar, extended minimum in solar activity also offers unique conditions to investigate the impact of solar, magnetospheric, and interplanetary phenomena in the Earth environment. Answering some of these challenges would positively contribute to expand our knowledge of the Earth atmospheric environment, namely of the coupling mechanisms between the troposphere, the ionosphere, and beyond.

\section{Acknowledgements}

FS and JK are supported by an appointment to the NASA Postdoctoral Program at the Goddard Space Flight Center, administered by Oak Ridge Associated Universities through a contract with NASA.

\section{References}

M. Abbas, Planet. Space Sci. 16, 831-844 (1968)

P.M. Adler, J.L. Le Mouel, J. Zlotnicki, Geophys. Res. Lett. 26, 795-798 (1999)

K. Aizawa, A. Yokoo, W. Kanda, Y. Ogawa, M. Iguchi, Geophys. Res. Lett. 37, L17301 (2010). doi: 10.1029/2010GL044208

W. Allan, E.M. Poulter, Rep. Prog. Phys. 55, 533-598 (1992)

W. Allan, A.N. Wright, J. Geophys. Res.-Space 102, 19927-19933 (1997)

R. Anderson, S. Björnsson, D.C. Blanchar et al., Science 148, 1179-1189 (1965)

Y. Ando, M. Hayakawa, A.V. Shvets, A.P. Nickolaenko, Radio Sci. 40, RS2002 (2005). doi:

10.1029/2004RS003153

S.D. Baker, M.C. Kelley, C.M. Swenson, J. Bonnell, D.V. Hahn, J. Atmos. Sol.-Terr. Phy. 62, 1393-1404 (2000)

L.M. Ball, Appl. Optics 13, 2292-2296 (1974)

M. Balser, C.A. Wagner, Nature 188, 638-641 (1960)

R. Barr, D.L. Jones, C.J. Rodger, J. Atmos. Sol.-Terr. Phy. 62, 1689-1718 (2000) 
T.L. Bell, D. Rosenfel, K.M. Kim, Geophys. Res. Lett., 36, L23805, (2009). doi: 10.1029/2009GL040915

P.P. Belyaev, T. Bösinger, S.V. Isaev, J. Kangas, J. Geophys. Res.-Space 104, 4305-4317 (1999)

P.P. Belyaev, S.V. Polyakov, V.O. Rapoport, V. Yu. Trakhtengerts, J. Atmos. Terr. Phys. 52, 781-788 (1990)

E.A. Bering, J.R. Benbrook, M.J. Engebretson, R.L. Arnoldy, J. Geophys. Res.-Space 103, 6741$6761(1998)$

J.-J. Berthelier, M. Malingre, R. Pfaff et al., Nat. Geosci. 1, 101-105 (2008)

C.J. Biagi, D.M. Jordan, M.A. Uman, J.D. Hill, W.H. Beasley, J. Howard, Geophys. Res. Lett. 36, L15801 (2009). doi: 10.1029/2009GL038525

J.W. Bieber, E. Eroshenko, P. Everson, E.O. Flückiger, R. Kallenbach, Cosmic rays and Earth (Space Science Reviews series 93, Kluwer Academic Publ, Dordrecht, Netherlands, 2000). ISBN 0-7923-6712-X

D. Bilitza, Radio Sci. 36, 261-275 (2001)

D. Bilitza, Adv. Space. Res. 44, 701-706 (2009)

J. Birn, J.F. Drake, M.A. Shay et al., J. Geophys. Res.-Space 106, 3715-3719 (2001)

C. Blagdon, Phil. Trans. Roy. Soc. 74, 201-232 (1784)

P.V. Bliokh, A.P. Nickolaenko, Yu.F. Filippov, in Schumann Resonances in the EarthIonosphere Cavity, ed.

by D.L. Jones (Peter Peregrinus, Oxford, 1980)

D.J. Boccippio, E.R. Williams, W.A. Lyons, I. Baker, R. Boldi, Science 269, 1088-1091 (1995)

D.J. Boccippio, C. Wong, E.R. Williams, R. Boldi, H.J. Christian, S.J. Goodman, J. Atmos. Sol.Terr. Phy. 60, 701-712 (1998)

J. Bortnik, U.S. Inan, T.F. Bell, J. Geophys. Res.-Space 111, A02204 (2006). doi: 10.1029/2005JA011182

T. Bösinger, A.G. Demekhov, V.Y. Trakhtengerts, Geophys. Res. Lett. 31, L18802 (2004). doi:10.1029/2004GL020777

T. Bösinger, S.L. Shalomov, Space Sci. Rev. 137, 521-532 (2008)

D.H. Brautigam, J.M. Albert, J. Geophys. Res.-Space 105, 291-309 (2000)

M. Brook, C.B. Moore, T. Sigurgei, J. Geophys. Res. 79, 472-475 (1974)

$\mathrm{K}$. Bullough, in Handbook of Atmospheric Electrodynamics II, 1st edn., ed. by H. Volland (CRC Press, Boca Raton, 1995), pp. 291-332

W.C. Burgess, U.S. Inan, J. Geophys. Res.-Space 98, 15,643-15,665 (1993)

L.B. Callis, D.N. Baker, J.B. Blake et al., J. Geophys. Res.-Atmos. 96, 2939-2976 (1991)

D.L. Carpenter, R.R. Anderson, J. Geophys. Res.-Space 97, 1097-1108 (1992)

D.L. Carpenter, U.S. Inan, Trimpi, M.L., R.A. Helliwell, J.P. Katsufrakis, J. Geophys. Res.Space 89, 9857-9862 (1984)

K.S. Carslaw, R.G. Harrison, J. Kirkby, Science 298, 1732-1737 (2002)

D.J. Cecil, E.J. Zipser, S.W. Nesbitt, Mon. Weather Rev. 130, 769-784 (2002)

Z. Ceplecha, J. Borovicka, W.G. Elford et al., Space Sci. Rev. 84, 327-471 (1998)

G. Chisham, D. Orr, J. Geophys. Res.-Space 102, 24,339-24,350 (1997)

Y.T. Chiu, B.C. Edgar, C.J. Rice, L.R. Sharp, Geophys. Res. Lett. 6, 455-458 (1979)

C. Christopoulos, The Transmission Line Modeling Method: TLM (IEEE Press, Piscataway, NY, 1995)

C.A. Colpitts, J. LaBelle, C.A. Kletzing, P.H. Yoon, J. Geophys. Res.-Space 115, A10243

(2010). doi: 10.1029/2009JA015095 
J.D. Colvin, C.K. Mitchell, J.R. Greig, D.P. Murphy, R.E. Pechacek, M. Raleigh, J. Geophys. Res. 92, 5696-5712 (1987)

N.F. Cramer, The Physics of Alfvén Waves (Wiley-VCH, Berlin, 2001)

R. Cross, An Introduction to Alfvén Waves (Adam Hilger Series on Plasma Physics, Taylor \& Francis, London, 1988)

S.A. Cummer, IEEE Antenn. Propag. 45, 392-400 (1997)

S.A. Cummer, U.S. Inan, T.F. Bell, Radio Sci. 33, 1781-1792 (1998)

S.A. Cummer, J.B. Li, F. Han et al., Nat. Geosci. 2, 617-620 (2009)

I.A. Daglis, R.M Thorne, W. Baumjohann, S. Orsini, Rev. Geophys. 37, 407-438 (1999)

R.E. Daniell, Jr., L. D. Brown, D. N. Anderson, et al., Radio Sci. 30, 1499-1510 (1995)

C.J. Davis, C.G. Johnson, Nature 435, 799-801 (2005)

O. de La Beaujardière and the C/NOFS Science Definition Team, J. Atmos. Solar-Terr. Phys. 66, 1573-1591 (2004). doi: 10.1016/j.jastp.2004.07.030.

O. de La Beaujardière, J. M. Retterer, R. F. Pfaff et al., Geophys. Res. Lett. 36, L00C06 (2009).

doi: 10.1029/2009GL038884.

A.G. Demekhov, V.Y. Trakhtengerts, T. Bösinger, Geophys. Res. Lett. 27, 3805-3808 (2000)

X.H. Deng, H. Matsumoto, Nature 410, 557-560 (2001)

A.J. Dessler, Nature 184, 261-262 (1959a)

A.J. Dessler, J. Geophys. Res. 64, 397-401 (1959b)

L.I. Dorman, Cosmic Rays in Magnetospheres of the Earth and Other Planets (Astrophysics and Space Science Library, Heidelberg, 2009)

R.L. Dowden, C.D.D. Adams, J.B. Brundell, P.E. Dowden, J. Atmos. Terr. Phys. 56, 1513-1527 (1994)

U. Ebert, S. Nijdam, C. Li, A. Duque, T. Briels, E. van Veldhuizen, J. Geophys. Res.-Space 115, A00E43 (2010). doi: 10.1029/2009JA014867

B.G. Elmegreen, J. Scalo, Annu. Rev. Astron. Astr. 42, 211-273 (2004)

M.J. Engebretson, L.J. Cahill, R.L. Arnold et al., J. Geophys. Res.-Space 96, 1527-1542 (1991)

W.M. Farrell, M.L. Kaiser, M.D. Desch et al., J. Geophys. Res.-Planet. 104, 3795-3801 (1999)

C. Ferencz, O.E. Ferencz, D. Hamar, J. Lichtenberger, Whistler Phenomena - Short Impulse

Propagation (Kluwer Academic Publishers, Dordrecht, 2001)

C.C. Finlay, S. Maus, C.D. Beggan et al., Geophys. J. Int. 183, 1216-1230 (2010)

L.A. Fisk, J.R. Jopikii, G.M. Simnett, R. von Steiger, K.-P. Wenzel, Cosmic Rays in the

Heliosphere (Space Science Reviews series 83, Kluwer Academic Publ, Dordrecht, Netherlands, 1998). ISBN 0-7923-5069-3

S.E. Forbush, Phys. Rev. 51, 1108-1109 (1937)

S.E. Forbush, Phys. Rev. 54, 975-988 (1938)

S.E. Forbush, J. Geophys. Res. 63, 651-669 (1958)

J.W. Fitzgerald, Atmos. Environ. A-Gen. 25, 533-545 (1991)

J.C. Foster, J.M. Holt, R.G. Musgrove, Geophys. Res. Lett. 13, 656-659 (1986)

A.V. Frank-Kamenetsky, L.N. Makarova, V.N. Morozov, A.V. Shirochkov, G.B. Burns, J.

Atmos. Sol.-Terr. Phy. 72, 419-424 (2010)

R.A. Frahm, J.D. Winningham, J.R. Sharber et al., J. Geophys. Res.-Atmos. 102, 28,203-28,214 (1997)

A.C. Fraser-Smith, Geophys. Res. Lett. 20, 467-470 (1993)

A.C. Fraser-Smith, A. Bernardi, P.R. McGill, M.E. Ladd, R.A. Helliwell, O.G. Villard, Geophys.

Res. Lett. 17, 1465-1468 (1990) 
M. Füllekrug, J. Atmos. Sol.-Terr. Phy. 66, 1115-1119 (2004)

M. Füllekrug, A.C. Fraser-Smith, Geophys. Res. Lett. 23, 2773-2776 (1996)

M. Füllekrug, M.J. Rycroft, Earth Planet. Space 58, 1193-1196 (2006)

J. Galejs, Terrestrial Propagation of Long Electromagnetic Waves (Pergamon, New York, 1972)

W. Gegelman, J. Geophys. Res.-Space 104, 14417-14435 (1999)

M.B. Gokhberg, V.A. Morgounov, T. Yoshino, I. Tomizawa, J. Geophys. Res. 87, 7824-7828 (1982)

M.V. Goldman, M.M. Oppenheim, D.L. Newman, Geophys. Res. Lett. 26, 1821-1824 (1999)

C. Greifinger, P.S. Greifinger, J. Geophys. Res. 73, 7473-7490 (1968)

C. Greifinger, P. Greifinger, J. Geophys. Res. 78, 4611-4618 (1973)

C. Greifinger, P. Greifinger, Radio Sci. 13, 831-837 (1978)

P.K.F. Grieder, Cosmic Rays at Earth: researcher's Reference Manual and Data Book (Elsevier Science, Amsterdam, 2001)

M. Grzesiak, Geophys. Res. Lett. 27, 923-926 (2000)

A.V. Gurevich, K.P. Zybin, Phys.-Usp.+44, 1119-1140 (2001)

D.A. Gurnett, S.D. Shawhan, N.M. Brice, R.L. Smith, J. Geophys. Res. 70, 1665-1688 (1965)

M. Gustafsson, S. He, Radio Sci. 35, 525-536 (2000)

C. Haldoupis, T. Neubert, U.S. Inan, A. Mika, T.H. Allin, R.A. Marshall, J. Geophys. Res.-Space 109, A10303 (2004). doi: 10.1029/2004JA010651

H.J. Hansen, B.J. Fraser, F.W. Menk, Y.D. Hu, P.T. Newell, C.I. Meng, Planet. Space Sci. 39, 709-719 (1991)

R.G. Harrison, Atmos. Environ. 36, 159-160 (2002)

R.G. Harrison, Surv. Geophys. 25, 441-484 (2004)

R.G. Harrison, K.L. Aplin, M.J. Rycroft, J. Atmos. Sol.-Terr. Phy. 72, 376-381 (2010)

R.G. Harrison, F. Märcz, Geophys. Res. Lett. 34, L23816 (2007). doi: 10.1029/2007GL031714

M. Hayakawa, A.P. Nickolaenko, M. Sekiguchi, K. Yamashita, Y. Ida, M. Yano, Nat. Hazard.

Earth Sys. 8, 1309-1316 (2008)

M. Hayakawa, K. Ohta, K. Baba, J. Geophys. Res. 99, 10,733-10,743 (1994)

M. Hayakawa, K. Ohta, A.P. Nickolaenko, Y. Ando, Ann. Geophys. 23, 1335-1346 (2005)

M. Hayakawa, T. Yoshino, V.A. Morgounov, Phys. Earth Planet. In. 77, 97-108 (1993)

S.L. He, P. Fuks, G.W. Larson, IEEE Antenn. Propag. 44, 1277-1282 (1996)

R.A. Helliwell, Whistlers and Related Ionospheric Phenomena (Stanford University Press,

Stanford, 1965)

R.H. Holzworth, M.C. Kelley, C.L. Siefring, L.C. Hale, J.D. Mitchell, J. Geophys. Res.-Space 90, 9824-9830 (1985)

R.B. Horne, M.M. Lam, J.C. Green, Geophys. Res. Lett. 36, L19104 (2009). doi:

10.1029/2009GL040236

R.B. Horne, R.M. Thorne, Nature 437, 227-230 (2005)

R.B. Horne, R.M. Thorne, S.A. Glauert, J.M. Albert, N.P. Meredith, R.R. Anderson, J. Geophys.

Res.-Space 110, A03225 (2005). doi: 10.1029/2005JA011516

E. Huang, E. Williams, R. Boldi et al., J. Geophys. Res.-Atmos. 104, 16,943-16,964 (1999)

J.D. Huba, G. Joyce, J.A. Fedder, J. Geophys. Res. 105, 23,035-23,053 (2000)

J.D. Huba, G. Joyce, S. Sazykin, R. Wolf, R. Spiro, Geophys. Res. Lett. 32, L23101 (2005). doi: 10.1029/2005GL024162

W.J. Hughes, D.J. Southwood, J. Geophys. Res. 81, 3234-3240 (1976) 
R.D. Hunsucker, J. K. Hargreaves, The High-Latitude Ionosphere and its Effects on Radio Propagation (Cambridge University Press, Cambridge, 2002)

W.L. Imhof, J.B. Reagan, H.D. Voss et al., Geophys. Res. Lett. 10, 361-364 (1983)

W.L. Imhof, J.B. Reagan, H.D. Voss et al., Geophys. Res. Lett. 10, 615-618 (1983)

U.S. Inan, S.A. Cummer, R.A. Marshall, J. Geophys. Res.-Space 115, A00E36 (2010). doi: 10.1029/2009JA014775

U.S. Inan, M. Golkowski, M.K. Casey et al., Geophys. Res. Lett. 34, L02106 (2007). doi: 10.1029/2006GL028494

T. Iyemori, M. Nose, D.S. Han et al., Geophys. Res. Lett. 32, L20807 (2005). doi:

10.1029/2005GL024083

C.H. Jackman, R.D. McPeters, G.J. Labow, E.L. Fleming, C.J. Praderas, J.M. Russell, Geophys.

Res. Lett. 28, 2883-2886 (2001)

R.P. James, P.M. Markowski, Mon. Weather Rev. 138, 140-161 (2010)

M.R. James, L. Wilson, S.J. Lane et al., Space Sci. Rev. 137, 399-418 (2008)

J. Jerauld, V.A. Rakov, M.A. Uman et al., J. Geophys. Res.-Atmos. 110, D19106 (2005). doi: 10.1029/2005JD005924

M.J.S. Johnston, Phys. Earth Planet. In. 57, 47-63 (1989)

M.J.S. Johnston, Surv. Geophys. 18, 441-476 (1997)

T.D. Kaladze, O.A. Pokhotelov, H.A. Shah, M.I. Khan, L. Stenflo, J. Atmos. Sol.-Terr. Phy. 70, 1607-1616 (2008)

A.K. Kamra, Nature 240, 143-144 (1972a)

A.K. Kamra, J. Geophys. Res. 77, 5856-5869 (1972b)

J. Kangas, A. Guglielmi, O. Pokhotelov, Space Sci. Rev. 83, 435-512 (1998)

J. Kasparian, R. Ackermann, Y.-B. André et al., Opt. Express 16, 5757-5763 (2008)

O. Katahira, Unzen. Res. Lett. Atmos. Electr. 12, 225-234 (1992)

C.S.L. Keay, Science 210, 11-15 (1980)

M.C. Kelley, The Earth's Ionosphere: Plasma Physics and Electrodynamics, (Academic Press, Oxford, 2009)

M.C. Kelley, D.T. Farley, E. Kudeki, C.L. Siefring, Geophys. Res. Lett. 11, 1168-1171 (1984)

M.C. Kelley, R. Livingston, M. McCready, Geophys. Res. Lett. 12, 577-580 (1985)

A. Khain, N. Cohen, B. Lynn, A. Pokrovsky, J. Atmos. Sci. 65, 3652-3677 (2008)

N. Khan, N. Mariun, I. Aris, J. Yeak, New J. Phys. 4, 61 (2002)

J. Kirkby, Surv. Geophys. 28, 333-375 (2007)

Y. Kito, K. Horii, Y. Higashiyama, K. Nakamura, J. Geophys. Res.-Atmos. 90, 6147-6157 (1985)

J.H. Klenzing, D.E. Rowland, R.F. Pfaff et al., J. Geophys. Res.-Space (2011).

doi:10.1029/2011JA016711, in press

J. Krall, J.D. Huba, C.R. Martinis, Geophys. Res. Lett. 36, L10103 (2009). doi: 10.1029/2009GL038441

P.R. Krehbiel, J.A. Riousset, V.P. Pasko, et al., Nat. Geosci. 1, 233-237 (2008)

S. Kumar, A. Kishore, V. Ramachandran, Ann. Geophys. 26, 1451-1459 (2008)

J. LaBelle, R.A. Treumann, Space Sci. Rev. 101, 295-440 (2002)

M.M. Lam, R.B. Horne, N.P. Meredith, S.A. Glauert, T. Moffat-Griffin, J.C. Green, J. Geophys.

Res.-Space 115, A00F08 (2010). doi: 10.1029/2009JA014619

J. Lastovicka, J. Atmos. Terr. Phys. 58, 831-843 (1996)

C.P. Liao, J.P. Freidberg, M.C. Lee, J. Atmos. Terr. Phys. 51, 751-758 (1989) 
D.R. Lide et al., CRC Handbook of Chemistry and Physics, 90th edn. (Taylor and Francis, Boca Raton, 2010)

W.A. Lyons, T.E. Nelson, E.R. Williams, J.A. Cramer, T.R. Turner, Science 282, 77-80 (1998)

R. L. Lysak, J. Geophys. Res.-Space 104, 10,017-10,030 (1999)

R. L. Lysak, J. Geophys. Res.-Space 109, A07201 (2004). doi:10.1029/2004JA010454

D.R. MacGorman, D.W. Burgess, Mon. Weather Rev. 122, 1671-1697 (1994)

T. Madden, W. Thompson, Rev. Geophys. 3, 211-254 (1965)

T.A. Mather, R.G. Harrison, Surv. Geophys. 27, 387-432 (2006)

S. Maus, M. Rother, C. Stolle et al., Geochem. Geophys. Geosy. 7, Q07008 (2006). doi:

10.1029/2006GC001269

V. Mazur, J. Geophys. Res.-Atmos. 94, 3326-3340 (1989)

J.C. McConnell, J.J. Jin, Atmos. Ocean 46, 69-92 (2008)

S.R. McNutt, C.M. Davis, J. Volcanol. Geoth. Res. 102, 45-65 (2000)

A.L. Mellot, D. Atri, B.C. Thomas, M.V. Medvedev, G.W. Wilson, M.J. Murray, J. Geophys.

Res.-Planet. 115, E08002 (2010). doi: 10.1029/2010JE003591

N.P. Meredith, M. Cain, R.B. Horne, R.M. Thorne, D. Summers, R.R. Anderson, J. Geophys.

Res.-Space 108, A61248 (2003). doi: 10.1029/2002JA009764

N.P. Meredith, R.B. Horne, R.R. Anderson, J. Geophys. Res.-Space 106, 13,165-13,178 (2001)

G. Milikh, R. Roussel-Dupré, J. Geophys. Res.-Space 115, A00E60 (2010). doi:

10.1029/2009JA014818

G.H. Millward, I.C.F. Müller-Wodarg, A.D. Aylward et al., J. Geophys. Res.-Space 106, 24,733-

25,744 (2001). doi: 10.1029/2000JA000342

L.I. Miroshnichenko, Solar Cosmic Rays (Kluwer Academic Publishers, Dordrecht, 2001)

A. Miura, Geophys. Res. Lett. 26, 409-412 (1999)

O.A. Molchanov, M. Hayakawa, J. Geophys. Res.-Space 103, 17,489-17,504 (1998)

V.C. Mushtak, E.R. Williams, J. Atmos. Sol.-Terr. Phy. 64, 1989-2001 (2002)

U. Narain, P. Ulmschneider, Space Sci. Rev. 75, 453-509 (1996)

D.A. Neudegg, B.J. Fraser, F.W. Menk, C.L. Waters, G.B. Burns, R.J. Morris, Adv. Space Res.

25, 1559-1565 (2000)

P.T. Newell, Y.I. Feldstein, Y.I. Galperin, C.I. Meng, J. Geophys. Res.-Space 101, 10,737$10,748(1996)$

P.T. Newell, C.I. Meng, Geophys. Res. Lett. 19, 609-612 (1992)

A.P. Nickolaenko, M. Hayakawa, Resonances in the Earth-Ionosphere Cavity (Kluwer

Academic Publishers, Dordrecht, 2002)

A.P. Nickolaenko, M. Hayakawa, Y. Hobara, J. Atmos. Terr. Phys. 58, 1699-1709 (1996)

A.P. Nickolaenko, M. Hayakawa, Y. Hobara, J. Geophys. Res.-Atmos. 104, 27585-27591 (1999)

C.A. Nucci, G. Diendorfer, M.A. Uman, F. Rachidi, M. Ianoz, C. Mazzetti, J. Geophys. Res.-

Atmos. 95, 20,395-20,408 (1990)

T. Ogawa, J. Geophys. Res. 90, 5951-5960 (1985)

H. Ohya, M. Nishino, Y. Murayama, K. Igarashi, A. Saito (2006), J. Atmos. Sol.-Terr. Phys. 68, 697- 709 (2006)

R.C. Olsen, D.M. Jordan, V.A. Rakov, M.A. Uman, N. Grimes, Geophys. Res. Lett. 31, L16107 (2004). doi: 10.1029/2004GL020187

J.V. Olson, J. Geophys. Res.-Space 104, 17499-17520 (1999)

A.A. Ostapenko, E.E. Titiva, A.P. Nickolaenko, T. Turunen, J. Manninen, T. Raita, Ann.

Geophys. 28, 193-202 (2010) 
A. Parent, I.R. Mann, I.J. Rae, J. Geophys. Res.-Space 115, A02312 (2010). doi: 10.1029/2009JA014673

C.G. Park, D.L. Carpenter, D.B. Wiggin, J. Geophys. Res.-Space 83, 3137-3144 (2008)

M. Parrot, in Handbook of Atmospheric Electrodynamics II, 1st edn., ed. by H. Volland (CRC Press, Boca Raton, 1995), pp. 95-116

V.P. Pasko, J. Geophys. Res.-Space 115, A00E35 (2010). doi: 10.1029/2009JA014860

V.P. Pasko, M.A. Stanley, J.D. Mathews, U.S. Inan, T.G Wood, Nature 416, 152-154 (2002)

F.W. Perkins, P. K. Kaw, J. Geophys. Res. 76, 282-284 (1971)

D. Petersen, M. Bailey, W.H. Beasley, J. Hallett, J. Geophys. Res.-Atmos. 113, D17205 (2008). doi: 10.1029/2007JD009036

J.M. Picone, A.E. Hedin, D.P. Drob, A.C. Aikin, J. Geophys. Res.-Space 107, A121468 (2002). doi: 10.1029/2002JA009430

G. Pfotzer, Z. Phys. 102, 23 (1936)

V.A. Pilipenko, J. Atmos. Terr. Phys. 52, 1193-1209 (1990)

S.V. Polyakov, V.O Rapoport, Geomag. Aeron. 21, 610-614 (1981)

C. Price, Nature 406, 290-293 (2000)

C. Price, M. Blum, Earth Moon Planets 82-83, 545-554 (2000)

C. Price, A. Melnikov, J. Atmos. Sol.-Terr. Phy. 66, 1179-1185 (2004)

V.A. Rakov, Uman, M.A., IEEE Electromagn. C. 40, 403-426 (1998)

V. Rakov, M. Uman, Lightning (Cambridge Univ. Press, Cambridge, 2007)

C.M. Rappaport, B.J. McCartin, IEEE Antenn. Propag. 39, 345-349 (1991)

O.M. Raspopov, L.J. Lanzerotti, Rev. Geophys. 14, 577-589 (1976)

J.B. Reagan, R.E. Meyerott, R.W. Nightingale et al., J. Geophys. Res.-Space 86, 1473-1494 (1981)

J.B. Reagan, T.M. Watt, J. Geophys. Res.-Space 81, 4579-4596 (1976)

D. Rees, J. Atmos. Terr. Phys. 57, 1433-1457 (1995)

C.D. Reeve, M.J. Rycroft, J. Atmos. Terr. Phys. 34, 667-672 (1972)

G.C. Reid, in Study in Geophysics: The Earth's Electrical Environment, ed. by E.P. Krider, R.G. Roble (National

Academy Press, Washington, 1986), pp. 183-194

N.O. Renno, J.F. Kok, Space Sci. Rev. 137, 419-434 (2008)

J.M. Retterer, D.T. Decker, W.S. Borer, R.E. Daniell Jr., B.G. Fejer, J. Geophys. Res. 110, A11307 (2005). doi:10.1029/2002JA009613

T. Rikitake, Y. Yamazaki, J. Geomagn. Geoelectr. 28, 497-505 (1976)

T. Rikitake, Y. Yamazaki, Earthquake Pred. Res. 3, 559-570 (1985)

B. Roberts, Sol. Phys. 193, 139-152 (2000)

R.G. Roble, B.A. Emery, T.L. Killeen et al., J. Geophys. Res.-Space 92, 6083-6090 (1987)

C.J. Rodger, M.A. Clilverd, R.J. McCormick, J. Geophys. Res.-Space 108, A121462 (2003). doi: 10.1029/2003JA009906

C.J. Rodger, M.A. Clilverd, N.R. Thomson, D. Nunn, J. Lichtenberger, Ann. Geophys. 23, 3419$3430(2005)$

C.J. Rodger, C.-F. Enell, E. Turenen, M.A. Clilverd, N.R. Thomson, P.T. Verronen, Ann.

Geophys. 25, 1745-1757 (2007)

J.V. Rodriguez, U.S. Inan, T.F. Bell, Geophys. Res. Lett. 19, 2067-2070 (1992a)

J.V. Rodriguez, U.S. Inan, Y.Q. Li, et al., J. Geophys. Res.-Space 97, 65-75 (1992b) 
V.C. Roldugin, Y.P. Maltsev, A.N. Vasiljev, A.V. Shvets, A.P. Nikolaenko, J. Geophys. Res.Space 108, A31103. doi:10.1029/2002JA009495 (2003)

M.J. Rycroft, J. Atmos. Sol.-Terr. Phy. 68, 445-456 (2006)

M.J. Rycroft, R.G. Harrison, K.A. Nicoll, E.A. Mareev, Space Sci. Rev. 137, 83-105 (2008)

M.J. Rycroft, S. Israelsson, C. Price, J. Atmos. Sol.-Terr. Phy. 62, 1563-1576 (2000)

M.J. Rycroft, A. Odzimek, N.F. Arnold, M. Fullekrug, A. Kulak, T. Neubert, J. Atmos. Sol.-

Terr. Phy. 69, 2485-2509 (2007)

S.V.S. Sarma, T.S. Sastry, J. Atmos. Terr. Phys. 57, 749-754 (1995)

G. Sátori, J. Atmos. Terr. Phys. 58, 1483-1488 (1996)

S.S. Sazhin, Whistler-Mode Waves in a Hot Plasma (Cambridge University Press, Cambridge, 1993)

S.S. Sazhin, M. Hayakawa, J. Atmos. Terr. Phys. 56, 735-753 (1994)

K. Schlegel, M. Füllekrug, J. Geophys. Res. 104, 10111-10118 (1999)

R. Schlickeiser, Cosmic Ray Astrophysics (Springer, Berlin, 2002)

W.O. Schumann, Z. Naturforschung A 7, 149-154 (1952) (in German)

U. Schumann, H. Huntrieser, Atmos. Chem. Phys. 7, 3823-3907 (2007)

R.W. Schunk, L. Scherliess, J.J. Sojka et al., Radio Sci. 39, RS1S02 (2004). doi

10.1029/2002RS002794

D.D. Sentman, J. Atmos. Terr. Phys. 52, 35-46 (1990)

D.D. Sentman, in Handbook of Atmospheric Electrodynamics I, 1st edn., ed. by H. Volland

(CRC Press, Boca Raton, 1995), pp. 267-298

D.D. Sentman, B.J. Fraser, J. Geophys. Res.-Space 96, 15,973-15,984 (1991)

S. Shalimov, M. Gokhberg, Phys. Earth Planet. In. 105, 211-218 (1998)

S.E. Sharapov, D. Testa, B. Alper et al., Phys. Lett. A 289, 127-134 (2001)

R.P. Sharma, M.L. Goldstein, N.K. Dwivedi, P.K. Chauhan, J. Geophys. Res.-Space 115, A12207 (2010). doi: 10.1029/2009JA015123

D.K. Sharma, J. Rai, M. Israil, P. Subrahmanyam, P. Chopra, S.C. Garg, Ann. Geophys. 22, 2047-2052 (2004)

M.A. Shay, J.F. Drake, B.N. Rodgers, R.E. Denton, J. Geophys. Res.-Space 106, 3759-3772 (2001)

Y.Y. Shprits, R.M. Thorne, Geophys. Res. Lett. 31, L08805 (2004). doi:

10.1029/2004GL019591

P.K. Shukla, L. Stenflo, Lect. Notes Phys. 536, 1-30 (1999)

A.B. Shvartsburg, L. Stenflo, J. Atmos. Terr. Phys. 55, 89-81 (1993)

A.V. Shvets, M. Hayakawa, J. Atmos. Sol.-Terr. Phy. 60, 461-469 (1998)

M. Stenhoff, Ball lightning: an unsolved problem in atmospheric physics (Kluwer Academic,

New York, 1999)

F. Simões, J.-J. Berthelier, M. Godefroy, S. Yahi, Geophys. Res. Lett. 36, L14816 (2009).

doi:10.1029/2009GL039286

F. Simões R. Grard, M. Hamelin et al., Planet. Space Sci. 55 1978-1989 (2007)

F. Simões, R. Grard, M. Hamelin et al., Icarus 194, 30-41 (2008c)

F. Simões, M. Hamelin, R. Grard et al., J. Geophys. Res.-Planet. 113, E07007 (2008b). doi:

10.1029/2007JE003045

F. Simões, R. Pfaff, H. Freudenreich, (submitted)

F. Simões, M. Rycroft, N. Renno, Y. Yair, K.L. Aplin, Y. Takahashi, Space Sci. Rev. 137, 455-

471 (2008a) 
J.J. Simpson, A. Taflove, IEEE Antenn. Propag. 55, 1582-1590 (2007)

A.K. Singh, R.P. Singh, Earth Moon Planets 73, 277-290 (1996)

D.K. Singh, R.P. Singh, A.K. Kamra, Space Sci. Rev. 113, 375-408 (2004)

D. Singh, R.P. Singh, A.K. Kamra et al., J. Atmos. Sol.-Terr. Phy. 67, 637-658 (2005)

T. Stanev, High Energy Cosmic Rays (Springer, Berlin, 2009)

K. Stasiewicz, P. Bellan, C. Chaston et al., Space Sci. Rev. 92, 423-533 (2000)

G.P. Stiller, G.M. Tsidu, T. von Clarmann et al., J. Geophys. Res.-Atmos. 110, D20303 (2005).

doi: 10.1029/2005JD006011

L.R.O. Storey, Philos. T. Roy. Soc. A 246, 113-141 (1953)

H.J. Strangeways, J. Geophys. Res.-Space 61, 1067-1080 (1999)

A.I. Sukhorukov, Ann. Geophys.-Atm. Hydr. 14, 33-41 (1996)

A.I. Sukhorukov, P. Stubbe, Geophys. Res. Lett. 24, 829-832 (1997)

D. Summers, C.Y. Ma, J. Geophys. Res.-Space 105, 2625-2639 (2000)

V.V. Surkov, M. Hayakawa, A.Y. Schekotov, E.N. Fedorov, O.A. Molchanov, J. Geophys. Res.Space 111, A01303 (2006). doi:10.1029/2005JA011320

V.V. Surkov, O.A. Molchanov, M. Hayakawa, E.N. Fedorov, J. Geophys. Res.-Space 110, A04308 (2005). doi: 10.1029/2004JA010850

K. Takahashi, Ann. Geophys.-Atm. Hydr. 16, 787-803 (1998)

R.W. Talbot, J.D. Bradshaw, S.T. Sandholm et al., J. Geophys. Res.-Atmos. 101, 24187-24202 (1996)

T. Tanaka, T. Ichinose, T. Okuzawa et al., J. Atmos. Terr. Phys. 46, 233-245 (1984)

M.J. Taylor, M.A. Hapgood, Planet. Space Sci. 36, 975-985 (1988)

B.J. Thompson, R.L. Lysak, J. Geophys. Res.-Space 101, 5359-5369 (1996)

B.A. Tinsley, Space Sci. Rev. 94, 231-258 (2000)

B.A. Tinsley, Rep. Prog. Phys. 71, 066801 (2008). doi: 10.1088/0034-4885/71/6/066801

B.A. Tinsley, G.B. Burns, L. Zhou, Adv. Space Res. 40, 1126-1139 (2007)

B.T. Tsurutani, W.D. Gonzalez, A.L.C. Gonzalez et al., J. Geophys. Res.-Space 111, A07S01

(2006). doi: 10.1029/2005JA011273

B.T. Tsurutani, C.M. Ho, Rev. Geophys. 37, 517-541 (1999)

M.A. Uman, D.F. Seacord, G.H. Price, E.T. Pierce, J. Geophys. Res. 77, 1591-1596 (1972)

I.G. Usoskin, L. Desorgher, P. Velinov et al., Acta Geophys. 57, 88-101 (2009)

H. Utada, T. Yoshino, T. Okubo, T. Yukutake, Tectonophysics 299, 317-331 (1998)

S. Uyeda, M. Hayakawa, T. Nagao et al., P. Natl. Acad. Sci. USA 99, $7352-7355$ (2002)

P.T. Verronen, A. Seppala, M.A. Clilverd et al., J. Geophys. Res.-Space 110, A09S32 (2005).

doi: 10.1029/2004JA010932

H.D. Voss, W.L. Imhof, M. Walt et al., Nature 312, 740-742 (1984)

J.W. Warwick, C. Stoker, T.R. Meyer, J. Geophys. Res. 87, 2851-2859 (1982)

E.R. Williams, Science 256, 1184-1187 (1992)

E.R. Williams, J. Geophys. Res.-Space 115, A00E50 (2010). doi: 10.1029/2009JA014581

E.R. Williams, V.C. Mushtak, R. Boldi, R.L. Dowden, Z.-I. Kawasaki, Radio Sci. 42, RS2S20

(2007). doi:10.1029/2006RS003498

E. Williams, K. Rothkin, D. Stevenson, D. Boccippio, J. Appl. Meteorol. 39, 2223-2230 (2000)

E.R. Williams, G. Satori, J. Atmos. Sol.-Terr. Phy. 66, 1213-1231 (2004)

C.T.R. Wilson, Proc. Camb. Philos. Soc. 13, 363-382 (1906)

C.T.R. Wilson, Phil.Trans. Roy. Soc. Lond. A 221, 73-115 (1920) 
J.H. Wolcott, D.J. Simons, D.D. Lee, R.A. Nelson, J. Geophys. Res.-Space 89, 6835-6839 (1984)

R.F. Woodman, E. Kudeki, Geophys. Res. Lett. 11, 1165-1167 (1984)

R.F. Woodman, C. Lahoz, J. Geophys. Res.-Space 81, 5447-5466 (1976)

N. Yagova, V. Pilipenko, E. Fedorov, M. Vellante, K. Yumoto, Earth Planets Space 51, 129-138 (1999)

Y. Yair, G. Fischer, F. Simões, N. Renno, P. Zarka, Space Sci. Rev. 137, 29-49 (2008)

A.G. Yahnin, T.A. Yahnina, J. Atmos. Sol.-Terr. Phy. 69, 1690-1706 (2007)

L. Yin, M. Ashour-Abdalla, Phys. Plasmas 6, 449-462 (1999)

J.L Young, Radio Sci. 29, 1513-1522 (1994)

Q.G. Zong, Y.Q. Hao, Y.F. Wang, Sci. China Ser. E 52, 3698-3708 (2009)

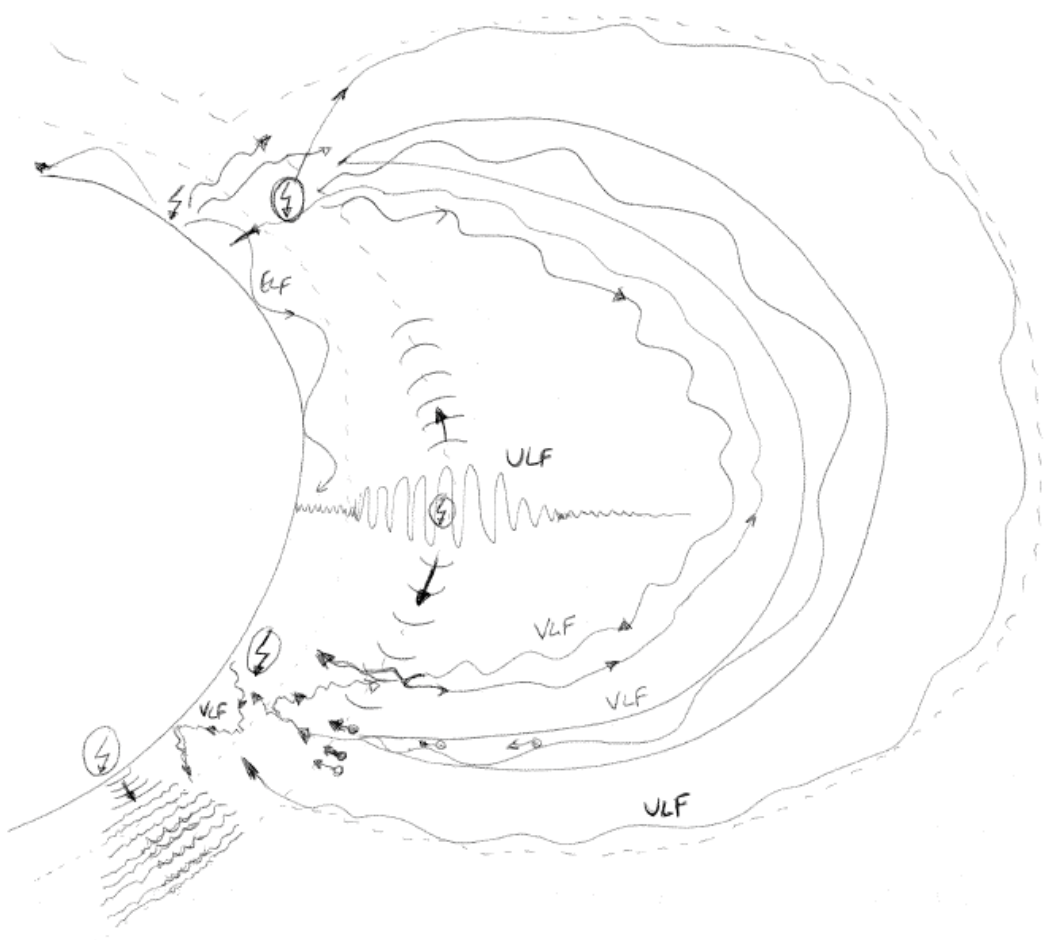

Figure 1: Sketch of ULF-VLF waves relevant for tropospheric-ionospheric coupling mechanisms investigation. (Partially inspired in the cover page of Handbook of atmospheric electrodynamics edited by Hans Volland. Sketch art quality will be improved.) 


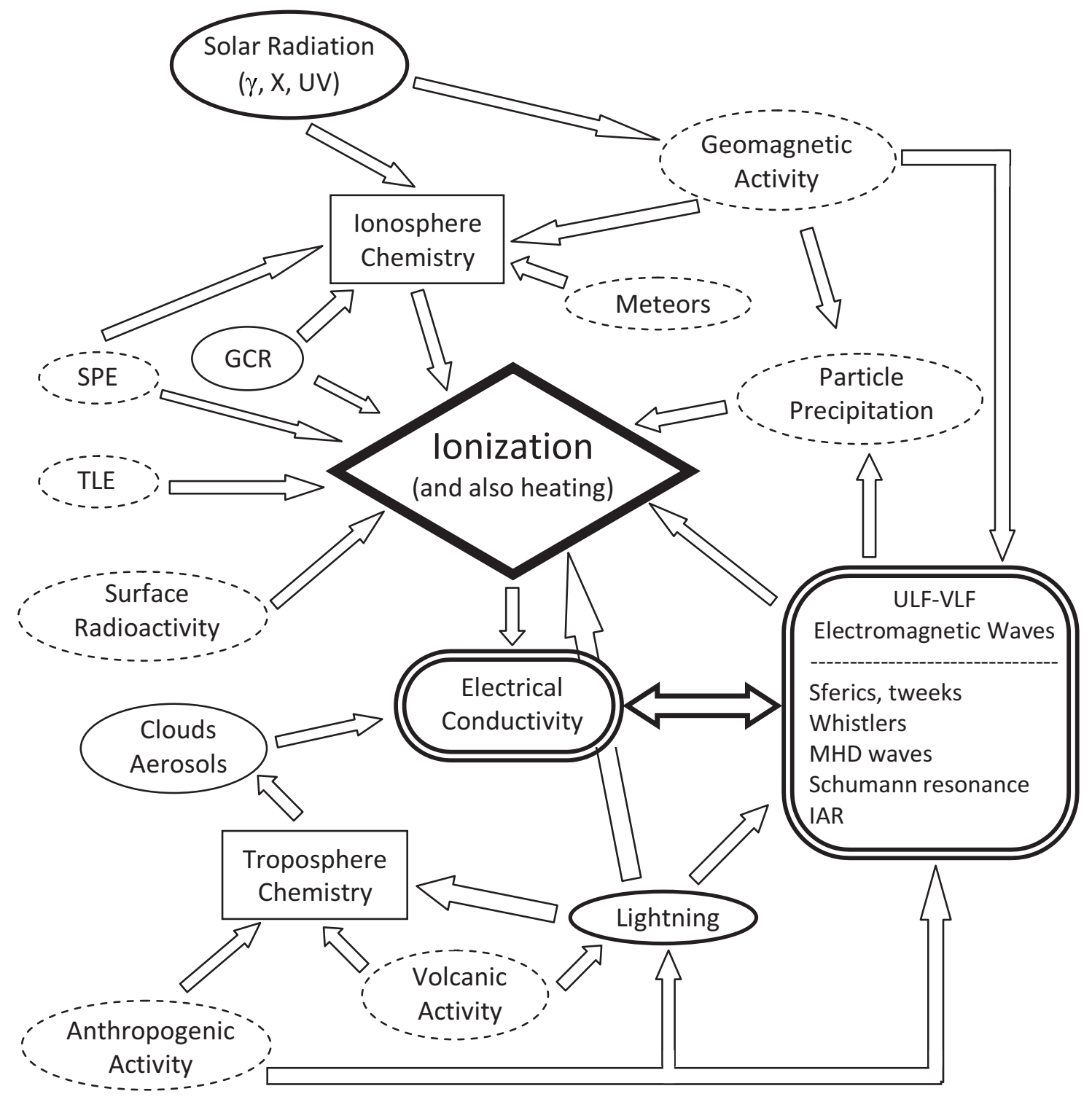

Figure 2: Diagram of sources that play a role in tropospheric-ionospheric coupling processes. Solid and dashed line ellipses represent permanent and transient activity prevalence, respectively. 